\title{
The Lindelöf property in Banach spaces
}

\author{
by \\ B. Cascales (Murcia), I. Namioka (Seattle, WA) and \\ J. Orihuela (Murcia)
}

\begin{abstract}
A topological space $(T, \tau)$ is said to be fragmented by a metric $d$ on $T$ if each non-empty subset of $T$ has non-empty relatively open subsets of arbitrarily small $d$-diameter. The basic theorem of the present paper is the following. Let $(M, \varrho)$ be a metric space with $\varrho$ bounded and let $D$ be an arbitrary index set. Then for a compact subset $K$ of the product space $M^{D}$ the following four conditions are equivalent:

(i) $K$ is fragmented by $d_{D}$, where, for each $S \subset D$,

$$
d_{S}(x, y)=\sup \{\varrho(x(t), y(t)): t \in S\} .
$$

(ii) For each countable subset $A$ of $D,\left(K, d_{A}\right)$ is separable.

(iii) The space $(K, \gamma(D))$ is Lindelöf, where $\gamma(D)$ is the topology of uniform convergence on the family of countable subsets of $D$.

(iv) $(K, \gamma(D))^{\mathbb{N}}$ is Lindelöf.

The rest of the paper is devoted to applications of the basic theorem. Here are some of them. A compact Hausdorff space $K$ is Radon-Nikodým compact if, and only if, there is a bounded subset $D$ of $C(K)$ separating the points of $K$ such that $(K, \gamma(D))$ is Lindelöf. If $X$ is a Banach space and $H$ is a weak ${ }^{*}$-compact subset of the dual $X^{*}$ which is weakly Lindelöf, then $(H, \text { weak })^{\mathbb{N}}$ is Lindelöf. Furthermore, under the same condition $\overline{\operatorname{span}(H)}\|\|$ and $\overline{\operatorname{co}(H)} w^{*}$ are weakly Lindelöf. The last conclusion answers a question by Talagrand. Finally we apply our basic theorem to certain classes of Banach spaces including weakly compactly generated ones and the duals of Asplund spaces.
\end{abstract}

1. Introduction. The starting point of the present investigation is a theorem by one of us in [25], namely that a Banach space $X$ is an Asplund space if and only if its dual $X^{*}$ is Lindelöf with respect to the topology of uniform convergence on bounded countable subsets of $X$, the $\gamma$-topology. In the present paper, we show that this result is a special case of a much more general theorem on function spaces and that it has interesting consequences including a solution to a question by Talagrand.

2000 Mathematics Subject Classification: Primary 46B99, 46A50; Secondary 54C35.

Key words and phrases: Lindelöf spaces, Radon-Nikodým compact spaces, fragmentability, $\gamma$-topology.

Partially supported by the research grant DGES PB 98-0381. 
This paper is organized as follows. After the introduction in Section 1, the basic theorem and its important corollary are stated and proved in Section 2.

In Section 3, a new characterization of Radon-Nikodým compact spaces by the Lindelöf property relative to the $\gamma$-topology is derived from the basic theorem. It will be shown that Meyer's characterization of compact scattered spaces [23] by the Lindelöf property with respect the $G_{\delta^{-}}$-topology is also a consequence of the basic theorem.

In Section 4, we use the Lindelöf property relative to the $\gamma$-topology to study the weakly Lindelöf property of sets in dual Banach spaces. We show, for instance, that the weak ${ }^{*}$-closed convex hull of a weak*-compact subset which is weakly Lindelöf in a dual Banach space is again weakly Lindelöf. This solves a problem of Talagrand in [32].

The theme of Section 4 is further expanded in Section 6 where it is proved, in particular, that the norm-closed linear span of a weak*-compact subset in a dual Banach space that is weakly Lindelöf is a WLD Banach space, as defined in Section 6. It should be noted here that each WLD Banach space is weakly Lindelöf and more. Our approach depends on the existence of "projectional generators" shown in Section 5. Our results on projectional generators also give a unified approach to the existence of projectional resolutions of the identity for both weakly compactly generated Banach spaces and duals of Asplund spaces.

In Section 7, we present several examples that illustrate the results of Sections 5 and 6 .

Our notation and terminology are standard and we take the books by Engelking and Kelley, [10] and [20], as our references for topology. Compact spaces are usually referred to by letters $K, H, \ldots$ and our normed spaces $X$, $Y, \ldots$ are assumed to be real. Given a topological space $Z$ we let $C(Z)$ (resp. $C_{\mathrm{b}}(Z)$ ) denote the space of real continuous (resp. real continuous uniformly bounded) functions defined on $Z$. For a Banach space $X, B_{X}$ denotes its closed unit ball and $X^{*}$ denotes its dual space. When $F$ is a subset of $X^{*}$, we write $\sigma(X, F)$ to denote the locally convex topology (maybe non-Hausdorff) on $X$ of pointwise convergence on $F ; \sigma\left(X, X^{*}\right)$ is the weak topology of $X$ and $\sigma\left(X^{*}, X\right)$ is the weak* topology of $X^{*}$. We consider $C_{\mathrm{b}}(Z)$ as a Banach space endowed with the supremum norm.

2. Fragmentability and the Lindelöf property for $\gamma(D)$. We first gather definitions of the terms and notation necessary for stating the main theorem of the present paper, Theorem 2.1. Recall that a topological space is said to be Lindelöf if each open cover of the space admits a countable subcover. The following definition is due to Jayne and Rogers [19]. 
Definition 1. Let $(Z, \tau)$ be a topological space and $\varrho$ a metric on $Z$. We say that $(Z, \tau)$ is fragmented by $\varrho$ (or $\varrho$-fragmented) if for each non-empty subset $C$ of $Z$ and for each $\varepsilon>0$ there exists a non-empty $\tau$-open subset $U$ of $Z$ such that $U \cap C \neq \emptyset$ and $\varrho$ - $\operatorname{diam}(U \cap C) \leq \varepsilon$.

It is easily checked that for $(Z, \tau)$ to be $\varrho$-fragmented, it is sufficient that each $\tau$-closed non-empty subset of $X$ has non-empty relatively $\tau$-open subsets of arbitrarily small $\varrho$-diameter.

Let $(M, \varrho)$ be a metric space and let $D$ be an arbitrary set. We shall write $\tau_{p}(D)$ (only $\tau_{p}$ if no ambiguity is likely) to denote the product topology of the space $M^{D}$. Assume henceforth that $\varrho$ is bounded, which can always be done without altering the uniformity of $M$. For any set $S \subset D$ we define the pseudo-metric $d_{S}$ on $M^{D}$ by the formula

$$
d_{S}(x, y)=\sup \{\varrho(x(t), y(t)): t \in S\} \quad \text { for } x, y \in M^{D} .
$$

The metric $d_{D}$ will be simply denoted by $d$; the topology associated to $d$ in $M^{D}$ is the topology of uniform convergence on $D$. Let $\gamma(D)$ denote the uniform topology on $M^{D}$ generated by the family of pseudo-metrics $\left\{d_{A}: A \subset D, A\right.$ countable $\}$, i.e. the topology of uniform convergence on the family of countable subsets of $D$.

The following notation is used in the proof of the next theorem. Let $2^{\mathbb{N}}$ be the space of all sequences of 0 's and 1's and let $2^{(\mathbb{N})}$ be the set of all finite sequences of 0 's and 1's. For a given $t \in 2^{(\mathbb{N})}$, let $|t|$ denote the length of $t$; for $\sigma \in 2^{\mathbb{N}}$ and $n \in \mathbb{N}$, we write $\sigma \mid n=(\sigma(1), \ldots, \sigma(n)) \in 2^{(\mathbb{N})}$.

Theorem 2.1. Let $(M, \varrho)$ and $D$ be as above, and let $K$ be a compact subset of $\left(M^{D}, \tau_{p}\right)$. Then the following conditions are equivalent:

(a) The space $\left(K, \tau_{p}\right)$ is fragmented by $d$.

(b) For each countable subset $A$ of $D,\left(K, d_{A}\right)$ is separable.

(c) The space $(K, \gamma(D))$ is Lindelöf.

Proof. (a) $\Rightarrow\left(\right.$ b). By Lemma 2.1 of $[24],\left(\left.K\right|_{A}, \tau_{p}(A)\right)$ is fragmented by $d_{A}$. Since $M^{A}$ is metrizable, $\left(\left.K\right|_{A}, \tau_{p}(A)\right)$ is compact metrizable; hence it has a countable base. If $\left(K, d_{A}\right)$ is not separable, then there is an uncountable subset $Q$ of $\left.K\right|_{A}$ and $\varepsilon>0$ such that $d_{A}(p, q)>\varepsilon$ whenever $p, q \in Q$ and $p \neq q$. We may assume that no point of $Q$ is $\tau_{p}$-isolated in $Q$ since $\left(\left.K\right|_{A}, \tau_{p}(A)\right)$ has a countable base. Since $\left(\left.K\right|_{A}, \tau_{p}(A)\right)$ is fragmented by $d_{A}$, there is a $\tau_{p}(A)$-open subset $U$ of $\left.K\right|_{A}$ such that $U \cap Q \neq \emptyset$ and $d_{A}$-diam $(U \cap Q)<\varepsilon$. Hence $U \cap Q$ is a singleton, contradicting the fact that no point of $Q$ is $\tau_{p}(A)$-isolated in $Q$. Hence $\left(K, d_{A}\right)$ is separable.

(b) $\Rightarrow(\mathrm{a})$. Suppose that $\left(K, \tau_{p}\right)$ is not fragmented by $d$. Then, for some non-empty $\tau_{p}$-closed subset $C$ of $K$ and $\varepsilon>0$, each non-empty $\tau_{p}$-open subset of $C$ has $d$-diameter greater that $\varepsilon$. By induction on $n=|s|, s \in 2^{(\mathbb{N})}$, we construct a family $\left\{U_{s}: s \in 2^{(\mathbb{N})}\right\}$ of non-empty relatively $\tau_{p}$-open subsets 
of $C$ and a family $\left\{t_{s}: s \in 2^{(\mathbb{N})}\right\}$ of points of $D$, satisfying the following conditions:

$(\alpha) U_{\emptyset}=C$.

(ß) $\bar{U}_{s 0}^{\tau_{p}} \cup \bar{U}_{s 1}^{\tau_{p}} \subset U_{s}$ for each $s$.

$(\gamma) \varrho\left(x\left(t_{s}\right), y\left(t_{s}\right)\right)>\varepsilon$ for each $x \in \bar{U}_{s 0}^{\tau_{p}}$ and $y \in \bar{U}_{s 1}^{\tau_{p}}$.

Construction. $(\alpha)$ starts the induction from $n=0$. Next, for some $n>0$, assume that $\left\{U_{s}:|s|<n\right\}$ and $\left\{t_{s}:|s|<n-1\right\}$ have been constructed. Fix an $s \in 2^{(\mathbb{N})}$ with $|s|=n-1$. By hypothesis, there are $x, y \in U_{s}$ with $d(x, y)>\varepsilon$. Hence for some $t_{s} \in D, \varrho\left(x\left(t_{s}\right), y\left(t_{s}\right)\right)>\varepsilon$. By the $\tau_{p}$-continuity of the map

$$
\left(x^{\prime}, y^{\prime}\right) \mapsto \varrho\left(x^{\prime}\left(t_{s}\right), y^{\prime}\left(t_{s}\right)\right)
$$

there are relatively $\tau_{p}$-open neighborhoods $U_{s 0}$ and $U_{s 1}$ of $x$ and $y$, respectively, so that $(\beta)$ and $(\gamma)$ are satisfied. This completes the construction. Note that $(\gamma)$ implies that $\bar{U}_{s 0}^{\tau_{p}} \cap \bar{U}_{s 1}^{\tau_{p}}=\emptyset$ for each $s \in 2^{(\mathbb{N})}$.

For each $\sigma \in 2^{\mathbb{N}}$, choose $x_{\sigma} \in \bigcap_{n=1}^{\infty} \bar{U}_{\sigma \mid n}^{\tau_{p}}$. If $\sigma, \sigma^{\prime} \in 2^{\mathbb{N}}$ are two different sequences, then for some $n \in\{0\} \cup \mathbb{N}, \sigma\left|n=\sigma^{\prime}\right| n$ and $\sigma\left|(n+1) \neq \sigma^{\prime}\right|(n+1)$. Then by $(\gamma)$ we have $\varrho\left(x_{\sigma}\left(t_{\sigma \mid n}\right), x_{\sigma^{\prime}}\left(t_{\sigma \mid n}\right)\right)>\varepsilon$. Letting $A=\left\{t_{s}: s \in 2^{(\mathbb{N})}\right\}$ we have $d_{A}\left(x_{\sigma}, x_{\sigma^{\prime}}\right)>\varepsilon$. Since $2^{\mathbb{N}}$ is uncountable, $\left(K, d_{A}\right)$ is not separable, and therefore (b) does not hold.

(c) $\Rightarrow(\mathrm{b})$. This is clear because the topology associated to $d_{A}$ is weaker than $\gamma(D)$ whenever $A$ is a countable subset of $D$.

(a) \&(b) $\Rightarrow(\mathrm{c})$. Let $\mathcal{U}=\left\{U_{j}: j \in J\right\}$ be a $\gamma(D)$-open cover of $K$ and let $\mathcal{C}=\{A: A \subset D$ and $A$ is countable $\}$. Without loss of generality we may assume that each $U_{j}$ is of the form

$$
U_{j}=U\left(x_{j}, A_{j}, \varepsilon_{j}\right):=\left\{y \in K: d_{A_{j}}\left(x_{j}, y\right)<\varepsilon_{j}\right\},
$$

where $x_{j} \in K, A_{j} \in \mathcal{C}$ and $\varepsilon_{j}>0$. For each $A \in \mathcal{C}$, define

$$
\mathcal{U}(A)=\left\{U_{j}: j \in J, A_{j} \subset A\right\} \quad \text { and } \quad U(A)=\bigcup\left\{U_{j}: U_{j} \in \mathcal{U}(A)\right\} .
$$

Then we have

$$
\mathcal{U}=\bigcup\{\mathcal{U}(A): A \in \mathcal{C}\} \quad \text { and } \quad K=\bigcup\{U(A): A \in \mathcal{C}\} .
$$

Also if $A \subset A^{\prime}$ then $U(A) \subset U\left(A^{\prime}\right)$.

We claim that $K=U(A)$ for some $A \in \mathcal{C}$. Suppose for a moment this is true. Then since each member of $\mathcal{U}(A)$ is $d_{A^{-}}$open and since $\left(K, d_{A}\right)$ is separable by (b), there is a countable subfamily of $\mathcal{U}(A)$ (hence of $\mathcal{U}$ ) that covers $K$, which completes the proof.

The proof of the claim is by contradiction. So assume that $U(A) \neq K$ for each $A \in \mathcal{C}$. For each $A \in \mathcal{C}$, let

$$
C(A)=K \backslash U(A) \quad \text { and } \quad C=\bigcap\left\{\overline{C(A)}^{\tau_{p}}: A \in \mathcal{C}\right\} .
$$


We note that $C(A) \supset C\left(A^{\prime}\right)$ whenever $A \subset A^{\prime}$. By compactness of $\left(K, \tau_{p}\right)$, $C \neq \emptyset$, and now (a) tells us that $\left(C, \tau_{p}\right)$ is fragmented by $d$. So by Lemma 1.1 of [24], there is a point $y \in C$ where the identity map $\left(C, \tau_{p}\right) \rightarrow(C, d)$ is continuous. The second equality in (2) ensures us that $y \in U(B)$ for some $B \in \mathcal{C}$. Since $U(B)$ is $d_{B}$-open, for some $\varepsilon>0, y \in U(y, B, \varepsilon) \subset U(B)$. Then for each $x \in C(B)=K \backslash U(B), x \notin U(y, B, \varepsilon)$ and so for some $t \in B$, $\varrho(x(t), y(t)) \geq 2 \varepsilon / 3$. For each $t \in B$, let

$$
D_{t}=\{x \in C(B): \varrho(x(t), y(t)) \geq 2 \varepsilon / 3\} .
$$

Then from the above, $C(B)=\bigcup\left\{D_{t}: t \in B\right\}$.

Let $V$ be a $\tau_{p}$-open neighborhood of $y$ in $K$ such that $d$-diam $\left(\bar{V}^{\tau_{p}} \cap C\right) \leq$ $\varepsilon / 2$. Then we claim that, for some $t \in B, D_{t} \cap V \cap C(A) \neq \emptyset$ for each $A \in \mathcal{C}$. For, otherwise, for each $t \in B$ there is an $A_{t} \in \mathcal{C}$ such that $D_{t} \cap V \cap C\left(A_{t}\right)=\emptyset$. Since $B$ is countable, the set $E:=B \cup \bigcup\left\{A_{t}: t \in B\right\}$ is also countable, and $D_{t} \cap V \cap C(E)=\emptyset$ for all $t \in B$. Hence

$$
\emptyset=\left(\bigcup\left\{D_{t}: t \in B\right\}\right) \cap V \cap C(E)=C(B) \cap V \cap C(E)=V \cap C(E),
$$

contradicting $y \in C \subset \overline{C(E)}^{\tau_{p}}$.

Now fix a $t \in B$ so that $D_{t} \cap V \cap C(A) \neq \emptyset$ for each $A \in \mathcal{C}$, and let

$$
z \in \bigcap\left\{{\overline{D_{t} \cap V \cap C(A)}}^{\tau_{p}}: A \in \mathcal{C}\right\} .
$$

Then $z \in \bar{V}^{\tau_{p}} \cap C$, and so

$$
d(z, y) \leq \varepsilon / 2 .
$$

On the other hand, since $z \in{\overline{D_{t}}}^{\tau_{p}}$, it follows by (3) that $\varrho(z(t), y(t)) \geq 2 \varepsilon / 3$, which contradicts (4). This completes the proof of both the claim and the theorem.

It is well known that the product of two Lindelöf spaces is not in general Lindelöf again: indeed, let $Z=\mathbb{R}$ and endow it with the topology for which a basis is given by all the intervals $[x, r)$, where $x, r \in \mathbb{R}, x<r$ and $r$ is a rational number; then $Z$ is a separable first-countable space that is Lindelöf and is not second-countable; moreover $Z \times Z$ is not normal and therefore not Lindelöf (see [10, pp. 248-249]).

Fortunately the Lindelöf property for the spaces $(K, \gamma(D))$ in Theorem 2.1 is preserved under the countable power.

Corollary 2.2. Let $K, M, D$ be as in Theorem 2.1. If $K$ satisfies one of the three conditions of the theorem, then $(K, \gamma(D))^{\mathbb{N}}$ is Lindelöf. In particular, $(K, \gamma(D))^{n}$ is Lindelöf for each $n \in \mathbb{N}$.

Proof. We may assume that the metric $\varrho$ of the space $M$ is bounded by 1 . Let $\varphi:\left(M^{D}\right)^{\mathbb{N}} \rightarrow\left(M^{\mathbb{N}}\right)^{D}$ be the map defined by $\varphi(\xi)(t)(j)=\xi(j)(t)$ for all $\xi \in\left(M^{D}\right)^{\mathbb{N}}, t \in D, j \in \mathbb{N}$. Clearly $\varphi$ is a homeomorphism when the 
product topology is used throughout. Now the space $M^{\mathbb{N}}$ is metrizable, and we use the metric $\varrho_{\infty}\left(m, m^{\prime}\right):=\sum_{j \in \mathbb{N}} 2^{-j} \varrho\left(m(j), m^{\prime}(j)\right)$ for $m, m^{\prime} \in M^{\mathbb{N}}$. Let $d_{\infty}$ be the metric on $\left(M^{\mathbb{N}}\right)^{D}$ given by

$$
d_{\infty}\left(x, x^{\prime}\right):=\sup \left\{\varrho_{\infty}\left(x(t), x^{\prime}(t)\right): t \in D\right\} \quad \text { for } x, x^{\prime} \in\left(M^{\mathbb{N}}\right)^{D} .
$$

We now show that if $K$ is fragmented by $d$ then $\varphi\left(K^{\mathbb{N}}\right)$ is fragmented by $d_{\infty}$. Let $\varepsilon>0$, let $C$ be a non-empty subset of $K^{\mathbb{N}}$ and let $\pi_{i}: K^{\mathbb{N}} \rightarrow K$ be the $i$-th projection. Then by induction we can construct a decreasing sequence $V_{1} \supset V_{2} \supset \ldots$ of non-empty relatively open subsets of $C$ such that $d$-diam $\left(\pi_{j}\left(V_{j}\right)\right)<\varepsilon / 2$ for each $j \in \mathbb{N}$. Choose $k \in \mathbb{N}$ so that $2^{-k}<\varepsilon / 2$, and let $\xi, \xi^{\prime} \in V_{k}$. Then for each $t \in D$,

$$
\begin{aligned}
\varrho_{\infty}\left(\varphi(\xi)(t), \varphi\left(\xi^{\prime}\right)(t)\right) & \leq \sum_{j \leq k} 2^{-j} \varrho\left(\xi(j)(t), \xi^{\prime}(j)(t)\right)+\sum_{j \geq k+1} 2^{-j} \\
& <\sum_{j \leq k} 2^{-j} d\left(\pi_{j}(\xi), \pi_{j}\left(\xi^{\prime}\right)\right)+\frac{\varepsilon}{2} \leq \frac{\varepsilon}{2}+\frac{\varepsilon}{2}=\varepsilon .
\end{aligned}
$$

Thus $\varphi\left(V_{k}\right)$ is a non-empty relatively open subset of $\varphi(C)$ with $d_{\infty}$-diameter not greater than $\varepsilon$.

Hence by Theorem 2.1, $\varphi\left(K^{\mathbb{N}}\right)$ is $\gamma(D)$-Lindelöf. So we finish the proof by showing that $\varphi$ maps $\left(M^{D}, \gamma(D)\right)^{\mathbb{N}}$ homeomorphically onto $\left(\left(M^{\mathbb{N}}\right)^{D}, \gamma(D)\right)$. Let $\tau_{1}, \tau_{2}$ be the topologies of these two spaces respectively. Then a net $\xi_{\alpha}$ in $\left(M^{D}\right)^{\mathbb{N}} \tau_{1}$-converges to $\xi \in\left(M^{D}\right)^{\mathbb{N}}$ if and only if: (i) for each $j \in \mathbb{N}$ and for each countable set $A \subset D, \varrho\left(\xi_{\alpha}(j)(t), \xi(j)(t)\right) \rightarrow 0$ uniformly in $t \in A$. On the other hand, the net $\varphi\left(\xi_{\alpha}\right) \tau_{2}$-converges to $\varphi(\xi)$ if and only if: (ii) for each countable $A \subset D$,

$$
\varrho_{\infty}\left(\varphi\left(\xi_{\alpha}\right)(t), \varphi(\xi)(t)\right)=\sum_{j \in \mathbb{N}} 2^{-j} \varrho\left(\xi_{\alpha}(j)(t), \xi(j)(t)\right) \rightarrow 0
$$

uniformly in $t \in A$. The equivalence of statements (i) and (ii) can be seen by an easy calculation similar to the one given above. Hence $\varphi$ is a $\tau_{1}-\tau_{2}$ homeomorphism.

As an immediate consequence of the foregoing, we obtain the following theorem, whose first part was mentioned in the introduction. It has been stated in [25] as Theorems B and C. The original proof is quite different and depends on the technique of projections in Banach spaces.

Theorem 2.3 ([25]). A Banach space $X$ is an Asplund space if and only if $\left(X^{*}, \gamma\left(B_{X}\right)\right)$ is Lindelöf. If this is the case, then $\left(X^{*}, \gamma\left(B_{X}\right)\right)^{n}$ is Lindelöf for each $n \in \mathbb{N}$.

Proof. Note that $\left(X^{*}, \gamma\left(B_{X}\right)\right)^{n}$ is Lindelöf if and only if $\left(B_{X^{*}}, \gamma\left(B_{X}\right)\right)^{n}$ is Lindelöf, and $X$ is an Asplund space if and only if $\left(B_{X^{*}}\right.$, weak $\left.{ }^{*}\right)$ is frag- 
mented by the norm. Therefore the theorem follows from 2.1 and 2.2 by regarding $\left(B_{X^{*}}\right.$, weak $\left.^{*}\right)$ as a compact subspace of $\left([-1,1]^{B_{X}}, \tau_{p}\right)$.

3. Applications to RN-compact spaces. Let $K$ be a compact Hausdorff space and let $D$ be a uniformly bounded subset of $C(K)$ and $A \subset D$. Then we define the pseudo-metric on $K$ by

$$
d_{A}\left(x, x^{\prime}\right)=\sup \left\{\left|f(x)-f\left(x^{\prime}\right)\right|: f \in A\right\} \quad \text { for } x, x^{\prime} \in K .
$$

We again write $\gamma(D)$ to denote the uniform topology on $K$ generated by the family of pseudo-metrics $\left\{d_{A}: A \subset D, A\right.$ countable $\}$. Observe that when $D$ separates the points of $K$, then $K$ embeds in $[-m, m]^{D}$ for some $m>0$. Hence the topology $\gamma(D)$ now defined is the one already given through the embedding $K \subset[-m, m]^{D}$, and $\gamma(D)$ is stronger than the original topology of $K$. In particular the equivalences we have seen in Theorem 2.1 and Corollary 2.2 remain true.

THEOREM 3.1. Let $K$ be a compact Hausdorff space and let $D$ be a uniformly bounded subset of $C(K)$. Then the following statements are equivalent:

(i) The space $\left(K, d_{A}\right)$ is separable for each countable $A \subset D$.

(ii) The space $(K, \gamma(D))$ is Lindelöf.

(iii) The space $(K, \gamma(D))^{\mathbb{N}}$ is Lindelöf.

Proof. From the remark above, the theorem is clear in case $D$ separates the points of $K$. The general case can be reduced to this as follows. Let $m=\sup \{\|f\|: f \in D\}$ and let $\varphi: K \rightarrow[-m, m]^{D}$ be the map given by $\varphi(x)(f)=f(x)$ for all $x \in K$ and $f \in D$. Then $K^{\prime}:=\varphi(K)$ is a compact Hausdorff space. For each $f \in D$, let $\widehat{f} \in C\left(K^{\prime}\right)$ be the map given by $\widehat{f}(\varphi(x))=f(x)$, and, for each $A \subset D$, let $\widehat{A}=\{\widehat{f}: f \in A\}$. Then clearly $f \mapsto \widehat{f}$ is a one-to-one map of $D$ onto $\widehat{D}$ and $d_{A}(x, y)=d_{\widehat{A}}(\varphi(x), \varphi(y))$ for all $x, y \in K$. It follows that $\left(K, d_{A}\right)$ is separable if, and only if, $\left(K^{\prime}, d_{\widehat{A}}\right)$ is separable. The last equality also implies that, for each $x \in K,\{y \in K$ : $\left.d_{A}(x, y)<\varepsilon\right\}=\varphi^{-1}\left(\left\{z \in K^{\prime}: d_{\widehat{A}}(\varphi(x), z)<\varepsilon\right\}\right)$. Hence a subset $U$ of $K$ is $\gamma(D)$-open if and only if $U=\varphi^{-1}\left(U^{\prime}\right)$ for some $\gamma(\widehat{D})$-open subset $U^{\prime}$ of $K^{\prime}$. From this it is straightforward to check that $(K, \gamma(D))\left(\operatorname{resp} .(K, \gamma(D))^{\mathbb{N}}\right)$ is Lindelöf if, and only if, $\left(K^{\prime}, \gamma(\widehat{D})\right)$ (resp. $\left.\left(K^{\prime}, \gamma(\widehat{D})\right)^{\mathbb{N}}\right)$ is Lindelöf. Since $\widehat{D}$ separates the points of $K^{\prime}$, the conclusion of the theorem is true for $\widehat{D}$ and $K^{\prime}$. Hence the theorem is proved in general.

A compact Hausdorff space is said to be Radon-Nikodým compact (or $\mathrm{RN}$-compact) if it is homeomorphic to a weak*-compact subset of the dual of an Asplund space, i.e. a dual Banach space with the RNP. It is shown in [24] that a compact Hausdorff space is RN-compact if, and only if, it is 
fragmented by a lower semicontinuous metric on the space. When $(M, \varrho)$ is a metric space (with $\varrho$ bounded) the metric $d$ in Theorem 2.1 is clearly $\tau_{p}$ lower semicontinuous. Therefore, Theorem 2.1 provides the following characterization of RN-compact spaces.

Proposition 3.2. A compact Hausdorff space is RN-compact if, and only if, it is homeomorphic to a pointwise compact subset $K$ of $[-1,1]^{D}$ for some set $D$ such that $(K, \gamma(D))$ is Lindelöf.

Proof. By Theorem 3.6 of [24] a compact space is RN-compact if, and only if, $K$ is homeomorphic to a pointwise compact subset $K$ of $[-1,1]^{D}$ for some set $D$ such that $\left(K, d_{A}\right)$ is separable for each countable subset $A$ of $D$. An application of Theorem 2.1 finishes the proof of the proposition.

In terms of spaces of continuous functions the proposition above can be restated as follows.

Corollary 3.3. A compact Hausdorff space $K$ is $R N$-compact if, and only if, there is a bounded subset $D$ of $C(K)$ separating points of $K$ such that $(K, \gamma(D))$ is Lindelöf. Moreover, if this is the case, then $(K, \gamma(D))^{\mathbb{N}}$ is Lindelöf.

Proof. Assume $K$ is RN-compact. By Proposition 3.2, we may assume that $K$ is a subspace of $\left([-1,1]^{D}, \tau_{p}\right)$ for a certain set $D$ with $(K, \gamma(D))$ Lindelöf; for every $d \in D$ let $\pi_{d}:[-1,1]^{D} \rightarrow[-1,1]$ be the projection defined by $\pi_{d}(x)=x(d), x \in[-1,1]^{D}$. If we let $\widehat{D}=\left\{\pi_{d}: d \in D\right\}$, then $\widehat{D}$ is a uniformly bounded subset of $C(K)$ separating the points of $K$ and such that $(K, \gamma(\widehat{D}))$ is Lindelöf. The last part follows from Theorem 3.1. A similar argument proves the converse.

For weakly compact subsets of $C(K)$, we have the following.

Corollary 3.4. Let $K$ be a compact Hausdorff space and let $H \subset C(K)$ be a weakly compact (i.e. bounded and $\tau_{p}$-compact) set. Then $(K, \gamma(H))^{\mathbb{N}}$ is Lindelöf.

Proof. For a countable set $A \subset H, \bar{A}^{\tau_{p}} \subset C(K)$ is $\tau_{p}(K)$-metrizable and thus the space $\left(C\left(\bar{A}^{\tau_{p}}\right), d_{\bar{A}^{\tau_{p}}}\right)$ is separable. Hence, $\left(\left.K\right|_{\bar{A}} \tau_{p}, d_{\bar{A}^{-}} \tau_{p}\right)$ is separable and so is $\left(K, d_{A}\right)$. In view of Theorem 3.1, the proof is complete.

We need the following easy lemma that appears in [5] in a more general context.

LEMma 1. Let $Z$ be a Lindelöf space, and let $H \subset C(Z)$ be equicontinuous. Then $\left(H, \tau_{p}(Z)\right)$ is metrizable.

Proof. Let $d_{H}$ be the pseudo-metric on $Z$ given by

$$
d_{H}\left(z, z^{\prime}\right)=\min \left\{1, \sup _{h \in H}\left|h(z)-h\left(z^{\prime}\right)\right|\right\} .
$$


Since $H$ is equicontinuous, the $d_{H}$-topology is weaker than the given one on $Z$. So $\left(Z, d_{H}\right)$ is Lindelöf and hence separable. Let $D$ be a countable $d_{H^{-}}$ dense subset of $Z$. Then since $H$ is $d_{H}$-equicontinuous, on $H$ the topologies of pointwise convergence on $D$ and on $Z$ coincide. Therefore $\left(H, \tau_{p}(Z)\right)$ is metrizable.

Given a subset $D$ of $\mathbb{R}^{K}$, let

$$
F(D)=\bigcup\left\{\bar{A}^{\tau_{p}}: A \subset D, A \text { countable }\right\} .
$$

Note that if $B$ is a countable subset of $F(D)$ then there is a countable subset $A$ of $D$ such that $\bar{B}^{\tau_{p}} \subset \bar{A}^{\tau_{p}} \subset F(D)$. In particular, $F(F(D))=$ $F(D)$.

Recall that a topological space $Z$ is said to be countably tight (resp. to be a Fréchet-Urysohn space) if for each set $S \subset Z$ and each point $x \in \bar{S}$ there is a countable set $A \subset S$ (resp. sequence $\left(x_{n}\right)_{n}$ in $S$ ) such that $x \in \bar{A}$ (resp. $\left(x_{n}\right)_{n}$ converges to $x$ ); see [2, pp. 5 and 7]. In applying the results of the last section, the following theorem of Arkhangel'skiu ([2, Theorem II.1.1]) is very useful. We quote a special case.

Theorem A. Let $T$ be a topological space such that $T^{n}$ is Lindelöf for each $n \in \mathbb{N}$. Then $\left(C(T), \tau_{p}(T)\right)$ is countably tight.

Corollary 3.5. Let $K$ be a compact space and let $D$ be a bounded subset of $C(K)$ such that $(K, \gamma(D))$ is Lindelöf. Then the following properties hold:

(a) For any countable set $A \subset D, \bar{A}^{\tau_{p}}$ (closure taken in $\mathbb{R}^{K}$ ) is $\gamma(D)$ equicontinuous and $\tau_{p}$-metrizable.

(b) $F(D)=C(K, \gamma(D)) \cap \bar{D}^{\tau_{p}}$, where the closure is taken in $\mathbb{R}^{K}$.

(c) $\left(F(D), \tau_{p}\right)$ is a Fréchet-Urysohn space.

Proof. (a) easily follows from the previous lemma: if $A \subset D$ is countable then $A$ is $\gamma(D)$-equicontinuous; its $\tau_{p}$-closure $\bar{A}^{\tau_{p}}$ in $\mathbb{R}^{K}$ is again $\gamma(D)$ equicontinuous and therefore $\tau_{p}$-metrizable by Lemma 1 . This proves (a).

For (b), we first note that (a) implies $F(D) \subset C(K, \gamma(D)) \cap \bar{D}^{\tau_{p}}$. Next we note that $(K, \gamma(D))^{n}$ is Lindelöf for each $n \in \mathbb{N}$ by Theorem 3.1. This fact implies that $\left(C(K, \gamma(D)), \tau_{p}\right)$ is countably tight according to Theorem A. Therefore if $f \in C(K, \gamma(D)) \cap \bar{D}^{\tau_{p}}$ then there is a countable subset $A$ of $D$ such that $f \in \bar{A}^{\tau_{p}}$. Hence $f \in F(D)$, which proves (b).

The proof of (c) is similar: Suppose that $S \subset F(D)$ and $f \in \bar{S}^{\tau_{p}} \cap F(D)$. Then by the countable tightness, there is a countable subset $B$ of $S$ such that $f \in \bar{B}^{\tau_{p}}$. Then as noted above, there is a countable subset $A$ of $D$ such that $\bar{B}^{\tau_{p}} \subset \bar{A}^{\tau_{p}}$. In particular $\bar{B}^{\tau_{p}}$ is $\tau_{p}$-metrizable by (a). Therefore there is a sequence in $B$ (hence in $S$ ) that $\tau_{p}$-converges to $f$. This proves (c). 
Recall that a topological space $T$ is said to be scattered if each non-empty subset of $T$ has an isolated point, or equivalently $T$ is fragmented by the (necessarily lower semicontinuous) trivial metric $\varrho$, where $\varrho(t, s)=0$ for $t=s$ and $\varrho(t, s)=1$ for $t \neq s$. It can be shown (cf. [30, Theorem 8.5.4]) that a compact Hausdorff space $K$ is scattered if and only if there is no continuous map from $K$ onto $[0,1]$. We remark that in the corollary above if $B_{C(K)} \subset F(D)$ then $K$ is scattered. For then, $\left(B_{C(K)}, \tau_{p}\right)$ is a FréchetUrysohn space; on the other hand, $\left(B_{C[0,1]}, \tau_{p}\right)$ is not Fréchet-Urysohn (see [2, Lemma II.3.5]), and consequently $K$ cannot be continuously mapped onto $[0,1]$.

Given a topological space $(Z, \mathcal{T})$, the $G_{\delta}$-topology associated to $\mathcal{T}$ is the topology on $Z$ whose basis is the family of $G_{\delta}$-sets, $\left\{\bigcap_{n} U_{n}: U_{n} \in \mathcal{T}\right\}$; when no confusion is likely we simply write $Z$ for the topological space and then refer to its $G_{\delta}$-topology.

Lemma 2. Let $K$ be a compact Hausdorff space. Then the $G_{\delta}$-topology for $K$ is identical with $\gamma\left(B_{C(K)}\right)$ on $K$.

Proof. Clearly the $G_{\delta}$-topology is stronger than $\gamma\left(B_{C(K)}\right)$. Let $a \in K$, and let $G$ be a $G_{\delta^{-}}$set containing $a$. Then $G=\bigcap_{n=1}^{\infty} U_{n}$ where each $U_{n}$ is open in $K$. For each $n$, let $f_{n}$ be a continuous function $f_{n}: K \rightarrow[0,1]$ such that $f_{n}(a)=0$, and $\left.f_{n}\right|_{K \backslash U_{n}} \equiv 1$. Write $A=\left\{f_{n}: n \in \mathbb{N}\right\}$. Then $A$ is a countable subset of $B_{C(K)}$, and $x \in G$ whenever $d_{A}(a, x)<1$, i.e.

$$
a \in\left\{x \in K: d_{A}(a, x)<1\right\} \subset G .
$$

This shows that $\gamma\left(B_{C(K)}\right)$ is stronger than the $G_{\delta}$-topology and we are done.

Corollary 3.6 (Meyer, [23]). For a compact Hausdorff space K, let $\tau_{\delta}$ denote its $G_{\delta}$-topology. Then the following statements are equivalent:

(a) $K$ is scattered.

(b) $\left(K, \tau_{\delta}\right)$ is Lindelöf.

(c) $\left(B_{C_{\mathrm{b}}\left(K, \tau_{\delta}\right)}, \tau_{p}\right)$ is a Fréchet-Urysohn space.

Proof. For $(\mathrm{a}) \Leftrightarrow(\mathrm{b})$, regarding $K$ as a subset of $\left([-1,1]^{B_{C(K)}}, \tau_{p}\right)$, we apply Theorem 2.1. In this case the metric $d$ is twice the trivial metric and the topology $\gamma\left(B_{C(K)}\right)$ is the $G_{\delta}$-topology for $K$ by the lemma above. $(\mathrm{a}) \Leftrightarrow(\mathrm{b})$ now follows. Next assume (b), and we apply Corollary 3.5 to our $K$ and $D:=B_{C(K)}$. The hypotheses are satisfied by (b). Since the $\tau_{p}$-closure of $D$ is $[-1,1]^{K}$, (b) of Corollary 3.5 says that $F(D)=B_{C_{\mathrm{b}}(K, \gamma(D))}=B_{C_{\mathrm{b}}\left(K, \tau_{\delta}\right)}$ and (c) of the same corollary says that $\left(B_{C_{\mathrm{b}}\left(K, \tau_{\delta}\right)}, \tau_{p}\right)$ is a Fréchet-Urysohn space. This is (c). If (c) holds, then $\left(B_{C(K)}, \tau_{p}\right)$ is also a Fréchet-Urysohn space. But as remarked above, this implies (a). 
We should comment here that topological spaces for which $G_{\delta}$-sets are again open are called $P$-spaces. It is a very easy exercise to prove that if $Z$ is a Lindelöf $\mathrm{P}$-space then $Z^{n}$ is Lindelöf for $n \in \mathbb{N}$ and so $\left(C(Z), \tau_{p}\right)$ has countable tightness; it also follows from Lemma 1 that for such a $Z$ the separable subsets of $\left(C(Z), \tau_{p}\right)$ are metrizable, and hence $\left(C(Z), \tau_{p}\right)$ is Fréchet-Urysohn; see also [2]. Our argument also shows that, for $K$ compact and scattered, the space of all continuous functions on $K$ endowed with its $G_{\delta^{-}}$-topology is $B_{1}(K)$, the space of $\tau_{p}$-limits of sequences in $C(K)$, and that all classes of Baire functions on $K$ are the same [22].

\section{Pointwise Lindelöf subsets of spaces of continuous functions.}

Let $D$ be a dense subset of a compact Hausdorff space $K$ and let $H$ be a bounded $\tau_{p}(D)$-compact subset of $C(K)$. In this section, we investigate the $\tau_{p}(K)$-Lindelöf property of $H$ by means of the $\gamma(D)$-topology of the earlier sections. As application we prove the results mentioned in the introduction.

The following simple proposition enables us to extract information on $\left(H, \tau_{p}(K)\right)$ from that on $(H, \gamma(D))$.

Proposition 4.1. Let $K$ be a compact Hausdorff space, $D$ a dense subset of $K$ and $H$ a subset $C(K)$. If $H$ is $\tau_{p}(K)$-Lindelöf, then $\gamma(D)$ is stronger than $\tau_{p}(K)$ on $H$.

Proof. Let $f \in H, \varepsilon>0, x \in K$, and

$$
U=\{g \in H:|g(x)-f(x)|<\varepsilon\} .
$$

Then $U$ is a $\tau_{p}(K)$-open neighborhood of $f$ in $H$, and it is sufficient to show that $U$ is a $\gamma(D)$-neighborhood of $f$ in $H$. For each $d \in D$, let

$$
D_{d}=\{g \in H:|g(d)-f(d)| \leq \varepsilon / 2\} .
$$

If $g \in \bigcap\left\{D_{d}: d \in D\right\}$, then $|g(x)-f(x)| \leq \varepsilon / 2$ since $x \in \bar{D}$, and therefore $g \in U$. It follows that $\bigcap\left\{D_{d}: d \in D\right\} \subset U$. Since each $D_{d}$ is $\tau_{p}(K)$-closed and $H$ is $\tau_{p}(K)$-Lindelöf, there is a countable subset $A$ of $D$ such that already $\bigcap\left\{D_{d}: d \in A\right\} \subset U$, i.e. $\left\{g \in H: \sup _{d \in A}|g(d)-f(d)| \leq \varepsilon / 2\right\} \subset U$. Hence $U$ is a $\gamma(D)$-neighborhood of $f$ in $H$ and the proof is finished.

Corollary 4.2. Let $K$ be a compact Hausdorff space, $D$ a dense subset of $K$ and $H$ a bounded $\tau_{p}(D)$-compact subset of $C(K)$. If $\left(H, \tau_{p}(K)\right)$ is Lindelöf, then $\left(H, \tau_{p}(K)\right)^{\mathbb{N}}$ is Lindelöf.

Proof. If $H$ is $\tau_{p}(D)$-compact and $\tau_{p}(K)$-Lindelöf, then by [4, Theorem B], $H$ is fragmented by the supremum norm of $C(K)$, i.e. as a compact subset $H$ of $[-m, m]^{D}$ for a suitable $m, H$ is fragmented by $d$ in the notation of Theorem 2.1. According to Theorem 2.1 and Corollary 2.2, $(H, \gamma(D))^{\mathbb{N}}$ is Lindelöf. By Proposition 4.1, $\gamma(D)$ is stronger than $\tau_{p}(K)$ on $H$ and there- 
fore $\left(H, \tau_{p}(K)\right)^{\mathbb{N}}$ is Lindelöf because it is a continuous image of the Lindelöf space $(H, \gamma(D))^{\mathbb{N}}$.

In [2, Problem IV.11.11] Arkhangel'skiu asks the following question. Let $K$ be a compact Hausdorff space. If there exists a $\tau_{p}$-Lindelöf subset $H$ of $C(K)$ that separates the points of $K$, is $K$ countably tight? The next corollary is an answer to this question under a rather strong restriction on $H$.

Corollary 4.3. Let $K$ be a compact Hausdorff space, and $H$ a $\tau_{p}(K)$ Lindelöf bounded subset of $C(K)$ separating the points of $K$. If $H$ is $\tau_{p}(D)$ compact for some dense subset $D \subset K$, then $K$ is countably tight.

Proof. An application of Corollary 4.2 allows us to conclude that $\left(H, \tau_{p}(K)\right)^{n}$ is Lindelöf for $n \in \mathbb{N}$. Hence the space $\left(C\left(H, \tau_{p}(K)\right), \tau_{p}(H)\right)$ is countably tight by Theorem A. The space $K$ is homeomorphic to a subset of $C\left(H, \tau_{p}(K)\right)$ because $H$ separates the points of $K$, and so the proof is done.

If $X$ is a Banach space, then $B_{X^{* *}}$ is always assumed to have the weak ${ }^{*}$ topology $\left(=\sigma\left(X^{* *}, X^{*}\right)\right)$ unless other topology is specified. Also $X$ and $B_{X}$ are considered as subspace/subset of $X^{* *}$ and $B_{X^{* *}}$, respectively, by means of the canonical embedding. Thus $\left(X^{*}\right.$, weak $\left.^{*}\right)$ is a subspace of $\left(C\left(B_{X^{* *}}\right)\right.$, $\left.\tau_{p}\left(B_{X}\right)\right)$ and $\left(X^{*}\right.$, weak $)$ is a subspace of $\left(C\left(B_{X^{* *}}\right), \tau_{p}\left(B_{X^{* *}}\right)\right)$. For a subset $S$ of $X^{*}$, the weak and weak ${ }^{*}$ closures of $S$ are respectively denoted by $\bar{S}^{\mathrm{w}}$ and $\bar{S}^{\mathrm{w}^{*}}$. A particular case of Corollary 4.2 is the following:

Corollary 4.4. Let $X$ be a Banach space and let $H$ be a weak ${ }^{*}$-compact subset of $X^{*}$ which is weakly Lindelöf. Then $(H, \text { weak })^{\mathbb{N}}$ is Lindelöf.

The next result gives an affirmative answer to a question posed by Talagrand that appears in [32] as Problème 4.5.

TheOrem 4.5. Let $X$ be a Banach space and let $H$ be a weak*-compact subset of $X^{*}$ which is weakly Lindelöf. Then:

(a) $\overline{\operatorname{co}(H)} \mathrm{w}^{*}=\overline{\operatorname{co}(H)}\|\|$.

(b) $\overline{\operatorname{co}(H)}^{\mathrm{w}^{*}}$ is weakly Lindelöf.

Proof. If $H$ is a weak ${ }^{*}$-compact subset of $X^{*}$ which is also weakly Lindelöf, then $\left(H\right.$, weak $\left.^{*}\right)$ is fragmented by the dual norm by Corollary E in [4]. The equality in item (a) now follows from Theorem 2.3 in [24].

Let us prove (b). As noted in the proof of (a), $\left(H\right.$, weak $\left.^{*}\right)$ is fragmented by the norm. Therefore if we let $W=\overline{\operatorname{co}(H)}^{\mathrm{w}^{*}}$, then $W$ is weak*-compact and $\left(W\right.$ weak $\left.^{*}\right)$ is fragmented by the norm by [24, Theorem 2.5]. By embedding $W$ into $[-m, m]^{B_{X}}$ for a suitable $m>0$, we see that $\left(W, \gamma\left(B_{X}\right)\right)$ is Lindelöf by Theorem 2.1. Therefore the proof is finished once we show 
that $\gamma\left(B_{X}\right)$ is stronger than the weak topology on $W$, or equivalently each member $x^{* *}$ of $B_{X^{* *}}$ is continuous on $\left(W, \gamma\left(B_{X}\right)\right)$. So fix an element $x^{* *}$ in $B_{X^{* *}}$. By Corollary $4.4,(H \text {, weak })^{\mathbb{N}}$ is Lindelöf, and therefore, by Theorem A, $\left(C(H\right.$, weak $\left.), \tau_{p}(H)\right)$ is countably tight. Since $\left.B_{X}\right|_{H}$ is $\tau_{p}(H)$-dense in $\left.B_{X^{* *}}\right|_{H} \subset C(H$, weak $)$, there is a countable subset $A \subset B_{X}$ such that $\left.x^{* *}\right|_{H}$ is in the $\tau_{p}(H)$-closure of $\left.A\right|_{H}$. Let $G$ be the convex hull of $H$. Then by the linearity, $\left.x^{* *}\right|_{W}$ is in the $\tau_{p}(G)$-closure of $\left.A\right|_{W}$. By (a), $G$ is norm-dense in $W$ and $\left.B_{X^{* *}}\right|_{W}$ is an equicontinuous family of functions on $(W,\|\|)$. Hence $\tau_{p}(W)$ and $\tau_{p}(G)$ coincide on $\left.B_{X^{* *}}\right|_{W}$, and so $\left.x^{* *}\right|_{W}$ is in the $\tau_{p}(W)$-closure of $\left.A\right|_{W}$. Finally, $\left.A\right|_{W}$ is an equicontinuous family on $\left(W, \gamma\left(B_{X}\right)\right)$ and hence $\left.x^{* *}\right|_{W}$, being in the pointwise closure of $\left.A\right|_{W}$, is $\gamma\left(B_{X}\right)$-continuous on $W$.

REMARK. In the theorem above as well as in the next corollary, the weak*-closed convex hull of $H\left(=\overline{\mathrm{co}(H)}^{\mathrm{w}}{ }^{*}\right)$ can be replaced by the weak*closed absolutely convex hull of $H$. The proof is almost identical as above since [24, Theorem 2.5] is actually stated for the weak* ${ }^{*}$-closed absolute convex hull case.

Corollary 4.6. Let $X$ be a Banach space, $H$ a weak*-compact subset of $X^{*}$ and $W$ its weak ${ }^{*}$-closed convex hull. The following statements are equivalent:

(a) $(H$, weak) is Lindelöf.

(b) $(H, \text { weak })^{\mathbb{N}}$ is Lindelöf.

(c) $(W$, weak) is Lindelöf.

(d) $(W, \text { weak })^{\mathbb{N}}$ is Lindelöf.

Proof. The implications $(\mathrm{a}) \Rightarrow(\mathrm{b})$ and $(\mathrm{c}) \Rightarrow(\mathrm{d})$ both follow from Corollary 4.4. The implications $(\mathrm{b}) \Rightarrow(\mathrm{a}),(\mathrm{d}) \Rightarrow(\mathrm{c})$ and $(\mathrm{c}) \Rightarrow(\mathrm{a})$ are obvious. And finally, the implication $(\mathrm{a}) \Rightarrow(\mathrm{c})$ is Theorem 4.5 .

5. Banach spaces generated by $\mathbf{R N}$-compact subsets. If $X$ is either a weakly compactly generated Banach space or the dual of an Asplund space, then $X$ is generated by an $\mathrm{RN}$-compact subset in the weak or the weak* topology. We shall deal in this section with the class of Banach spaces generated by $\mathrm{RN}$-compact subsets with respect to a topology weaker than the weak topology. To be more concrete, our framework is the following: for a Banach space $(X,\|\|)$ we consider a norming subset $F \subset X^{*}$ (also called 1-norming subset) for $X$, that is, a $\mathbb{Q}$-linear set $F$ satisfying

$$
\|x\|=\sup \left\{|\langle x, f\rangle|: f \in F \cap B_{X^{*}}\right\} .
$$

If a bounded set $H \subset X$ is $\sigma(X, F)$-compact and fragmented by the norm, then $(H, \sigma(X, F))$ is an $\mathrm{RN}$-compact set since the norm is $\sigma(X, F)$-lower semicontinuous, and we will study the space generated by it, that is, the 
space $Y=\overline{\operatorname{span}(H)}\|\|$. The Banach space $Y$ thus obtained will be called a Banach space generated by an $R N$-compact subset. In Section 7 , we exhibit several examples of such Banach spaces. In order to show the main properties of spaces generated this way we shall first see that these spaces admit projectional generators as defined below. Here our main reference is [11]. If $A$ is a non-empty subset of a Banach space $X$, then $A^{\perp}$ denotes the subset $\left\{f \in X^{*}: f(x)=0\right.$ for all $\left.x \in A\right\}$ of $X^{*}$.

Definition 2. Let $X$ be a Banach space. A projectional generator on $X$ is a countable-valued map $\varphi: F \rightarrow 2^{X}$ on a norming subset $F \subset X^{*}$ such that whenever $B \subset F$ is a $\mathbb{Q}$-linear set, we have

$$
\varphi(B)^{\perp} \cap \overline{B \cap B_{X^{*}}} \mathrm{w}^{*}=\{0\}
$$

According to the method developed in [27], [25] and [11], the existence of a projectional generator leads to the existence of a projectional resolution of identity (PRI for short) in the sense that follows. Given a Banach space $X$, the density character of $X$ (denoted by dens $X$ ) is defined to be the least cardinality of a dense subset of $X$. Let $\mu$ be the least ordinal such that $|\mu|=$ dens $X$, where $|\mu|$ denotes the cardinality of the ordinal $\mu$. A PRI on $X$ is a transfinite sequence $\left\{P_{\alpha}: \omega_{0} \leq \alpha \leq \mu\right\}$ of linear projections in $X$ satisfying the following conditions, where $\alpha$ and $\beta$ are arbitrary ordinals in $\left[\omega_{0}, \mu\right]:$

(a) $\left\|P_{\alpha}\right\|=1$.

(b) dens $P_{\alpha}(X) \leq|\alpha|$.

(c) $P_{\alpha} P_{\beta}=P_{\beta} P_{\alpha}=P_{\min \{\alpha, \beta\}}$.

(d) For each $x \in X$ and each limit ordinal $\alpha, P_{\beta}(x) \rightarrow P_{\alpha}(x)$ in the norm as $\beta \uparrow \alpha$.

The next proposition gathers the main properties of spaces with a projectional generator. In what follows, "LUR norm" stands for "locally uniformly rotund (or convex) norm".

Each part of the following theorem is known, but they are not usually stated in the form we prefer in the present paper. We record it here for later reference.

TheOREM 5.1. Let $X$ be a Banach space with a projectional generator $\varphi: F \rightarrow 2^{X}$. Then the following statements hold:

(a) $X$ admits a PRI $\left\{P_{\alpha}: \omega_{0} \leq \alpha \leq \mu\right\}$ such that $P_{\alpha}(X)$ has a projectional generator for each $\omega_{0} \leq \alpha<\mu$.

(b) $X$ admits an equivalent LUR norm.

(c) There is a linear continuous one-to-one operator $T: X \rightarrow c_{0}(\Gamma)$ for some set $\Gamma$. 
(d) The Banach space $X$ is $\gamma(X, F)$-Lindelöf, where $\gamma(X, F)$ is the topology on $X$ of uniform convergence on bounded countable subsets of $F$.

Proof. (a) With the projectional generator $\varphi$ in $X$, a PRI $\left\{P_{\alpha}: \omega_{0} \leq \alpha\right.$ $\leq \mu\}$ can be constructed, based on pairs $\left(A_{\alpha}, B_{\alpha}\right)$ of $\mathbb{Q}$-linear subsets, $A_{\alpha} \subset$ $X$ and $B_{\alpha} \subset F$ with $\varphi\left(B_{\alpha}\right) \subset A_{\alpha}$ and $B_{\alpha}$ norming for $A_{\alpha}$ (see Proposition 6.1.7 and Remark 6.1.8 of [11]); so, we have $\overline{B_{\alpha} \cap B_{X^{*}}}{ }^{*} \cap A_{\alpha}^{\perp}=\{0\}$ and $P_{\alpha}$ is the projection from $X$ onto $\bar{A}_{\alpha}^{\|\|}$with kernel $B_{\alpha}^{\perp}$. The space $P_{\alpha}^{*}\left(X^{*}\right)=$ $\bar{B}_{\alpha}^{\mathrm{w}^{*}}$ is identified with the dual of $P_{\alpha}(X)=\bar{A}_{\alpha}^{\|\|}$and therefore $P_{\alpha}(X)$ also has a projectional generator defined on $B_{\alpha}$ by $\varphi_{\alpha}(f)=P_{\alpha}(\varphi(f)), f \in B_{\alpha}$. These observations complete the proof of (a).

(b) and (c). Here we use the induction argument encapsulated in [7, Theorem VII.1.8]. Let $\mathcal{P}$ be the class of Banach spaces that admit a projectional generator. Then (a) shows that the hypothesis on $\mathcal{P}$ in $[7$, Theorem VII.1.8] is satisfied. Therefore each member $X$ of $\mathcal{P}$ admits an equivalent LUR norm. If, in the proof of [7, Theorem VII.1.8], one uses [11, Proposition 6.2.2] instead of Proposition VII.1.6 of [7], then one can also conclude that each member $X$ of $\mathcal{P}$ has property (c).

(d) The proof of Theorem A in [25] gives us this result.

What remains of this section is devoted to proving that a Banach space generated by an $\mathrm{RN}$-compact subset has a projectional generator and therefore enjoys the properties listed in Theorem 5.1.

First we recall Simons' lemma [31].

LEMMA 3. Let $\left(z_{n}\right)_{n}$ be a uniformly bounded sequence in $\ell^{\infty}(C)$ and let $W$ be its convex hull. If $B$ is a subset of $C$ such that for every sequence $\left(\lambda_{n}\right)_{n}$ of positive numbers with $\sum_{n=1}^{\infty} \lambda_{n}=1$ there is $b \in B$ such that

$$
\sup \left\{\sum_{n=1}^{\infty} \lambda_{n} z_{n}(y): y \in C\right\}=\sum_{n=1}^{\infty} \lambda_{n} z_{n}(b),
$$

then

$$
\sup _{b \in B}\left\{\limsup _{n \rightarrow \infty} z_{n}(b)\right\} \geq \inf \left\{\sup _{C} w: w \in W\right\} .
$$

A subset of $X^{*}$ is said to be total if its linear span is weak*-dense in $X^{*}$. Clearly a norming subset for $X$ is a total subset of $X^{*}$.

Definition 3. Let $X$ be a normed space, $C \subset X$ a set and $F$ a total subset in $X^{*}$. A subset $B \subset C$ is said to be an $F$-boundary for $C$ if for every $f$ in $F$ there is a $b \in B$ such that $f(b)=\sup \{f(x): x \in C\}$.

In what follows, when $F$ is a total norm-closed subspace of $X^{*}$ we consider the norm associated to $F$ given by

$$
p_{F}(x)=\sup \left\{|\langle x, f\rangle|: f \in F \cap B_{X^{*}}\right\},
$$


for $x \in X$. Then the unit ball of $\left(X, p_{F}\right)^{*}$ is the set $\overline{F \cap B_{X^{*}}} w^{*}$ and $\left(X, p_{F}\right)^{*}$ is the subspace $H=\bigcup_{n=1}^{\infty} n \overline{\left(F \cap B_{X^{*}}\right)}$ w* of $X^{*}$. Clearly $F \subset H$.

Proposition 5.2. Let $X$ be a normed space and let $F$ be a total normclosed subspace of $X^{*}$. Let $C$ be a bounded subset of $X$ and $B \subset C$ an $F$-boundary for $C$ such that $\left(B, p_{F}\right)$ is separable. Then

$$
\overline{\operatorname{co}(B)}^{p_{F}}=\overline{\operatorname{co}(C)}^{\sigma(X, F)} \text {. }
$$

Proof. The proof is based on the ideas in [13] (see also [12]). As we remarked, the dual of $\left(X, p_{F}\right)$ is the subspace $H=\bigcup_{n=1}^{\infty} n \bar{G}^{\mathrm{w}^{*}}$ of $X^{*}$, where $G=B_{X^{*}} \cap F$, and $F \subset H$. Hence

$$
\overline{\operatorname{co}(B)}^{p_{F}} \subset \overline{\operatorname{co}(C)}^{p_{F}}=\overline{\operatorname{co}(C)}^{\sigma(X, H)} \subset \overline{\operatorname{co}(C)}^{\sigma(X, F)} .
$$

Assume that the conclusion of the proposition is false. Then there exists an element $x_{0} \in \overline{\operatorname{co}(C)}^{\sigma(X, F)} \backslash \overline{\operatorname{co}(B)}^{p_{F}}$. Then by the separation theorem, there is a functional $f \in H=\left(X, p_{F}\right)^{*}$ such that

$$
f\left(x_{0}\right)>\alpha>\sup \{f(b): b \in B\} .
$$

By scaling we may assume that $f \in \bar{G}^{\mathrm{w}^{*}}$. Let $U=\left\{g \in X^{*}: g\left(x_{0}\right)>\alpha\right\}$. Then $U$ is convex weak* ${ }^{*}$-open and $f \in \bar{G}^{\mathrm{w}^{*}} \cap U \subset \overline{G \cap U}^{\mathrm{w}^{*}}$. Now $\bar{G}^{\mathrm{w}^{*}}$ is equicontinuous on $\left(X, p_{F}\right)$ and $B$ contains a countable $p_{F}$-dense subset $D$. Therefore in $\bar{G}^{\mathrm{w}^{*}}$ the topology of pointwise convergence on $B$ is identical with the topology of pointwise convergence on $D$, and the latter is pseudometrizable. It follows that there is a sequence $\left\{z_{n}: n \in \mathbb{N}\right\}$ in $G \cap U$ such that $\lim _{n} z_{n}(b)=f(b)$ for each $b \in B$. Our assumption of $F$ being norm-closed and $B$ being an $F$-boundary of $C$ implies that the sequence $\left(z_{n}\right)_{n}$ satisfies the hypothesis of Lemma 3. Hence by (8),

$$
\alpha>\sup _{b \in B} f(b) \geq \inf \left\{\sup _{c \in C} w(c): w \in \operatorname{co}\left(\left\{z_{n}\right\}\right)\right\} .
$$

It follows that $\alpha>\sup _{C} w$ for some $w \in \operatorname{co}\left(\left\{z_{n}\right\}\right) \subset G \cap U$. In particular, since $w \in U, w\left(x_{0}\right)>\alpha>\sup _{C} w$. On the other hand, since $x_{0} \in \overline{\operatorname{co}(C)}^{\sigma(X, F)}$ and, being in $F, w$ is $\sigma(X, F)$-continuous, $w\left(x_{0}\right) \leq \sup _{C} w$, contradicting the previous inequality. This proves the proposition.

The pointwise limit of a sequence of real-valued continuous functions is called a function of the first Baire class. More generally a function $f$ from a topological space $M$ into a normed space $X$ is said to be of the first Baire class if there is a sequence of continuous functions $f_{n}: M \rightarrow X$ such that $\left(f_{n}\right)_{n}$ converges to $f$ in $\left(X^{M}, \tau_{p}\right)$. A multi-valued map $\varphi$ from the topological space $M$ to the space of subsets of a topological space $T$ is said to be usco if $\varphi(m)$ is a compact non-empty subset of $T$ for each $m \in M$ and if $\varphi$ is 
upper semicontinuous in the sense that, whenever $U$ is an open subset of $T$, $\{m \in M: \varphi(m) \subset U\}$ is open in $M$.

Ideas in [14] (see also [29]) allow us to modify Jayne-Rogers' selection theorem, [19], to our situation below.

TheOREM 5.3. Let $M$ be a metric space, $X$ a normed space and $F$ a total norm-closed subspace of $X^{*}$. Let $H$ be a norm-bounded $\sigma(X, F)$-compact subset of $X$ which is fragmented by the norm $p_{F}$. If $\psi$ is an usco map from $M$ to subsets of $(H, \sigma(X, F))$, then $\psi$ has a first Baire class selector $f$ from $M$ into $\left(X, p_{F}\right)$.

Proof. If we identify $\left(X, p_{F}\right)$ with a subspace of $\ell^{\infty}\left(F \cap B_{X^{*}}\right)$ and $H$ with a weak*-compact subset there, then we can apply Remark 17 in [18] to obtain a selector $f$ of $\psi_{H}$ which is $\sigma$-discrete and of the first Borel class from $F$ to $\ell^{\infty}\left(B \cap B_{X^{*}}\right)$ (see Corollary 7 in [18]). Such a selector as a map from $F$ into $\left(X, p_{F}\right)$ is also $\sigma$-discrete of the first Borel class, and by Theorems 1 and 2 of $[29], f$ is of the first Baire class from $F$ into $\left(X, p_{F}\right)$ (see also [14] and the remarks in the introduction of [29]).

We are now ready to prove one of the main properties of the selectors obtained above: the result that follows is a counterpart to the one stated as Theorem 26 in [18], and it is in the setting of topologies of pointwise convergence on total sets.

TheOREM 5.4. Let $X$ be a normed space and let $F$ be a total norm-closed subspace of $X^{*}$. Let $H$ be a norm-bounded $\sigma(X, F)$-compact subset of $X$. Let $\psi_{H}: F \rightarrow 2^{H}$ be the multi-valued map given by

$$
\psi_{H}(f)=\left\{x \in H: f(x)=\sup _{H} f\right\} .
$$

Then $\psi_{H}$ has a selector of the first Baire class from $(F,\|\|)$ into $\left(X, p_{F}\right)$ if, and only if,$(H, \sigma(X, F))$ is fragmented by $p_{F}$. Moreover, if $f: F \rightarrow H$ is such a selector of $\psi_{H}$, then

$$
\overline{\operatorname{co}(H)}^{\sigma(X, F)}=\overline{\operatorname{co}(f(F))}^{p_{F}} .
$$

Proof. The arguments here are similar to the ones in [18, Theorem 26]. First it is easy to check that $\psi_{H}$ is an usco map from $(F,\|\|)$ into compact subsets of $(H, \sigma(X, F))$. If $(H, \sigma(X, F))$ is fragmented by $p_{F}$, then, by Theorem 5.3, $\psi_{H}$ has a first Baire class selector $f:(F,\|\|) \rightarrow\left(X, p_{F}\right)$. Conversely assume that such a selector $f$ exists. Let $S$ be a \|\| -closed and \|\| -separable subspace of $F$, and consider the quotient normed space $\left(X / S^{\perp},\|\|_{S}\right)$. Recall that the dual of $\left(X / S^{\perp},\|\|_{S}\right)$ is isometric with $\bar{S}^{\mathrm{w}^{*}}$ and hence $S$ is a norm-closed total subspace of $\left(X / S^{\perp},\|\|_{S}\right)^{*}$. Let $\pi_{S}: X \rightarrow X / S^{\perp}$ be the canonical quotient map and let $p_{S}$ be the norm on $X / S^{\perp}$ given by

$$
p_{S}\left(\pi_{S}(x)\right)=\bar{p}_{S}(x):=\sup \left\{|g(x)|: g \in S \cap B_{X^{*}}\right\}
$$


for each $x \in X$. Then $\pi_{S}(H)$ is a \|\|$_{S^{-b o u n d e d}} \sigma\left(X / S^{\perp}, S\right)$-compact subset of $X / S^{\perp}$, and $\pi_{S}(f(S))$ is an $S$-boundary for $\pi_{S}(H)$. Now let $f_{k}: F \rightarrow X$ be a sequence of \|\|$-p_{F}$ continuous maps such that for each $g \in F, f_{k}(g) \rightarrow f(g)$ in $p_{F}$. For each subset $A$ of $F$, let

$$
\Phi(A)=\bigcup_{k=1}^{\infty} f_{k}(A) .
$$

Then $f\left(\bar{A}^{\|\|}\right) \subset \overline{\Phi(A)}^{p_{F}}$ and $\Phi(A)$ is countable whenever $A$ is. If $D$ is a \|\| dense countable subset of $S$, then $f(S)=f\left(\bar{D}^{\|\|}\right) \subset \overline{\Phi(D)}^{p_{F}}$. Hence $f(S)$ is $p_{F}$-separable and so $\pi_{S}(f(S))$ is $p_{S}$-separable. It follows from Proposition 5.2 that

$$
\overline{\operatorname{co}\left(\pi_{S}(f(S))\right)} p_{S}={\overline{\operatorname{co}\left(\pi_{S}(H)\right)}}^{\sigma\left(X / S^{\perp}, S\right)} .
$$

This shows in particular that, whenever $S$ is a $\|$ \|-separable \| \|-closed subspace of $F, \pi_{S}(H)$ is $p_{S^{-}}$-separable and hence $H$ is $\bar{p}_{S^{-}}$-separable. Regarding $H$ as a $\tau_{p^{-}}$-compact subset of $[-m, m]^{F \cap B_{X^{*}}}$ with an appropriate $m>0$, we see from Theorem 2.1 that $(H, \sigma(X, F))$ is fragmented by $p_{F}$.

Finally we show that (10) is a consequence of (12). For this it is sufficient to prove that for each $u \in X$, there is a \|\| -separable \|\| -closed subspace $S$ of $F$ such that

$$
p_{S}-\operatorname{dist}\left(\pi_{S}(u), \operatorname{co}\left(\pi_{S}(f(S))\right)\right) \geq p_{F^{-}} \operatorname{dist}(u, \operatorname{co}(f(S))) .
$$

For if $u \in \overline{\operatorname{co}(H)} \sigma(X, F)$ and if $S$ is chosen as above, then since

$$
\pi_{S}(u) \in \overline{\pi_{S}(\operatorname{co}(H))} \sigma\left(X / S^{\perp}, S\right)
$$

we have, by (12), $0=p_{S}$-dist $\left(\pi_{S}(u), \operatorname{co}\left(\pi_{S}(f(S))\right) \geq p_{F}\right.$-dist $(u, \operatorname{co}(f(S)))$. Hence $u \in \overline{\operatorname{co}(f(S))}^{p_{F}} \subset \overline{\operatorname{co}(f(F))} p_{F}$. This shows that the left side of (10) is contained in the right side. The reverse inclusion is obvious.

To prove (13), let $u \in X$. For each countable subset $M$ of $X$, let $\alpha(M)$ be a countable subset of $F \cap B_{X^{*}}$ such that, for each $x \in M$,

$$
p_{F}(u-x)=\sup \{|g(u-x)|: g \in \alpha(M)\} .
$$

Inductively we define a sequence $A_{1} \subset A_{2} \subset \ldots$ of countable subsets of $F$ as follows: let $g_{0}$ be an arbitrary non-zero element of $F$ and let $A_{1}=\left\{q g_{0}\right.$ : $q \in \mathbb{Q}\}$. Assuming that $A_{n}$ has been defined, let

$$
A_{n+1}=\operatorname{span}_{\mathbb{Q}}\left(\alpha\left(\cos _{\mathbb{Q}}\left(\Phi\left(A_{n}\right)\right)\right) \cup A_{n}\right),
$$

where $\operatorname{span}_{\mathbb{Q}}(C)$ (resp. $\operatorname{co}_{\mathbb{Q}}(C)$ ) denotes the set of all linear (resp. convex) combinations of elements of $C$ with rational coefficients. Let $S=$ $\overline{\bigcup_{n=1}^{\infty} A_{n}}\|\|$.

Before showing this $S$ satisfies (13), we note that if $y \in \operatorname{cog}_{\mathbb{Q}}\left(\Phi\left(A_{n}\right)\right)$ then $p_{F}(u-y)=\sup \left\{|g(u-y)|: g \in \alpha\left(\operatorname{co}_{\mathbb{Q}}\left(\Phi\left(A_{n}\right)\right)\right)\right\} \leq \bar{p}_{S}(u-y) \leq p_{F}(u-y)$. 
Hence $p_{F}(u-y)=\bar{p}_{S}(u-y)$. Now by the definition of $\Phi$,

$$
\begin{aligned}
\operatorname{co}(f(S)) & \subset \operatorname{co}\left(\overline{\Phi\left(\bigcup_{n=1}^{\infty} A_{n}\right)} p_{F}\right) \\
& \subset \overline{\operatorname{co}_{\mathbb{Q}}\left(\bigcup_{n=1}^{\infty} \Phi\left(A_{n}\right)\right)} p_{F}=\overline{\bigcup_{n=1}^{\infty} \operatorname{co}_{\mathbb{Q}}\left(\Phi\left(A_{n}\right)\right)} p_{F} .
\end{aligned}
$$

Let $x \in \operatorname{co}(f(S))$ and $\varepsilon>0$ be arbitrary. Then there is a $y \in \operatorname{co}_{\mathbb{Q}}\left(\Phi\left(A_{n}\right)\right)$ for some $n$ such that $\bar{p}_{S}(x-y) \leq p_{F}(x-y)<\varepsilon$. Then

$$
\begin{aligned}
p_{S}\left(\pi_{S}(u)-\pi_{S}(x)\right) & =\bar{p}_{S}(u-x) \geq \bar{p}_{S}(u-y)-\varepsilon=p_{F}(u-y)-\varepsilon \\
& \geq p_{F}(u-x)-2 \varepsilon \geq p_{F}-\operatorname{dist}(u, \operatorname{co}(f(S)))-2 \varepsilon .
\end{aligned}
$$

Since $x \in f(S)$ and $\varepsilon>0$ are arbitrary, we obtain (13).

REMARK. In the setting of Theorem 5.4, (12) is now true whenever $S$ is a \|\| -closed subspace of $F$. This can be seen by applying Theorem 5.4 to the normed space $X / S^{\perp}$, the total subspace $S$ of $\left(X / S^{\perp}\right)^{*}=\bar{S}^{\mathrm{w}^{*}}$, the $\sigma\left(X / S^{\perp}, S\right)$-compact set $\pi_{S}(H)$ and the selector $\left.\pi_{S} \circ f\right|_{S}$ for the usco map $\psi_{S}: S \rightarrow 2^{\pi_{S}(H)}$. This remark is important in the proof of the next theorem.

Theorem 5.5. Let $X$ be a Banach space, $F$ a norming subset of $X^{*}$, and let $H$ be a bounded $\sigma(X, F)$-compact subset of $X$ fragmented by the norm of $X$. Then the Banach space $Y=\overline{\operatorname{span}(H)}\|\|$ has a projectional generator.

Proof. We first prove the case $X=Y$. Since $H$ is bounded, $\sigma(X, F)$ and $\sigma\left(X, \bar{F}^{\|\|}\right)$coincide on $H$. Hence we may assume that $F$ is a \|\| closed norming subspace. Let $\psi_{H}: F \rightarrow 2^{H}$ be the set-valued map given by $\psi_{H}(g)=\left\{x \in H: g(x)=\sup _{H} g\right\}$ for each $g \in F$. Then by Theorem 5.4, $\psi_{H}$ admits a selector $f: F \rightarrow H$ of the first Baire class from $(F,\|\|)$ into $(X,\|\|)$. Let $\left\{f_{k}\right\}$ be a sequence of continuous maps $(F,\|\|) \rightarrow(X,\|\|)$ such that $f_{k}(g) \rightarrow f(g)$ in the norm for each $g \in F$, and we define the countable-valued map $\varphi: F \rightarrow 2^{X}$ by $\varphi(g)=\left\{f_{k}(g): k \in \mathbb{N}\right\}$. We prove that $\varphi$ is a projectional generator (cf. Definition 2). So let $B$ be a $\mathbb{Q}$-linear subset of $F$, and let $g \in \varphi(B)^{\perp} \cap \overline{B \cap B_{X^{*}}} \mathrm{w}^{*}$. We must show that $g=0$.

Let $S=\bar{B}^{\|\|} \subset F$, let $\pi_{S}: X \rightarrow X / S^{\perp}$ be the quotient map and let $p_{S}$ be the norm defined on $X / S^{\perp}$ by (11). Since $g \in \overline{S \cap B_{X^{*}}}{ }^{*}, g$ defines a $p_{S^{-}}$continuous linear functional $\bar{g}$ on $X / S^{\perp}$ by the formula $\bar{g}\left(\pi_{S}(x)\right)=g(x)$ for each $x \in X$. Now by the definition of $\varphi, f(S)=f\left(\bar{B}^{\|\|}\right) \subset \overline{\varphi(B)}\|\|$. Since $g$ vanishes on $\varphi(B)$, it also vanishes on $f(S)$, and hence $\bar{g}$ vanishes on $\pi_{S}(f(S))$. By the remark following the last theorem, (12) is valid for $S$ and hence $\pi_{S}(H) \subset \overline{\operatorname{co}\left(\pi_{S}(f(S))\right.} p_{S}$. Therefore by continuity $\bar{g}$ vanishes on $\pi_{S}(H)$, i.e. $g$ vanishes on $H$. Since $X$ is the norm-closed span of $H, g=0$. 
The general case is proved by applying the special case above to the Banach space $Y$ and the norming subspace $\left.F\right|_{Y}$ for $Y$. Note that $H$ is a $\sigma\left(Y,\left.F\right|_{Y}\right)$-compact subset of $Y$ and it is fragmented by the norm of $Y$.

Corollary 5.6. Let $X$ be a Banach space, $F$ a norming subset of $X^{*}$, $H$ a bounded subset of $X$ which is $\sigma(X, F)$-compact and fragmented by the norm of $X$, and let $Y=\overline{\operatorname{span}(H)}\|\|$. Then:

(a) $(Y, \gamma(X, F))$ is Lindelöf.

(b) $Y$ has a PRI.

(c) $Y$ has an equivalent LUR norm.

Proof. This is a straightforward consequence of Theorems 5.1 and 5.5.

Another property of spaces generated by RN-compact sets is the following. For this, we need one more definition. Let $(Z, \tau)$ be a topological space and $\varrho$ a metric on $Z$. Then $(Z, \tau)$ is said to be $\sigma$-fragmented by $\varrho$ if for each $\varepsilon>0, Z$ can be written as $Z=\bigcup\left\{Z_{n}: n \in \mathbb{N}\right\}$ with each $Z_{n}$ having the property that, whenever $C$ is a non-empty subset of $Z_{n}$, there exists a $\tau$-open subset $U$ of $Z$ such that $U \cap C$ is non-empty and of $\varrho$-diameter less than $\varepsilon$.

Theorem 5.7. Let $X$ be a Banach space, $F$ a norming subset of $X^{*}, H$ a bounded subset of $X$ which is $\sigma(X, F)$-compact fragmented by the norm of $X$, and let $Y=\overline{\operatorname{span}(H)}\|\|$. Then $(Y, \sigma(X, F))$ is $\sigma$-fragmented by the norm.

Proof. The proof is analogous to the one given for weakly compactly generated spaces in [15]. Indeed, $W:=\overline{\operatorname{co}(H)}^{\sigma(X, F)}=\overline{\operatorname{co}(H)}^{\|\|}$is $\sigma(X, F)$ compact and fragmented by the norm [6, 4.1, 5.2 and 5.3]. Lemmas 2.1 and 2.2 of [24] entail that $W-W$ is again $\sigma(X, F)$-compact and fragmented by the norm. We now have $Y={\overline{\bigcup_{n=1}^{\infty} n(W-W)}}^{\|\|}$and because $F$ is norming, the norm in $Y$ is $\sigma(X, F)$-lower semicontinuous and Lemma 2.3 in [15] gives us the conclusion.

We can gather all the information that we have obtained so far in the following:

Theorem 5.8. Let $X$ be a Banach space, $F$ a norming subset of $X^{*}, H$ a bounded subset of $X$ which is $\sigma(X, F)$-compact, and let $Y=\overline{\operatorname{span}(H)}\|\|$. The following statements are equivalent:

(a) $(H, \sigma(X, F))$ is fragmented by the norm.

(b) $(Y, \sigma(X, F))$ is $\sigma$-fragmented by the norm.

(c) $(H, \gamma(X, F))$ is Lindelöf.

(d) $(Y, \gamma(X, F))$ is Lindelöf. 
Proof. (a) $\Rightarrow$ (b) is the previous result. (b) $\Rightarrow$ (a) follows from Lemma 3.1.1 in [16]. (a) $\Rightarrow(d)$ is the item (a) of Corollary 5.6. (d) $\Rightarrow(c)$ is obvious and (c) $\Rightarrow$ (a) is also the implication (c) $\Rightarrow($ a) in Theorem 2.1 .

In terms of compact sets embedded in cubes, the theorem above can be rephrased as:

Theorem 5.9. Let $K \subset[-1,1]^{D} \subset \ell^{\infty}(D)$ be a $\tau_{p}$-compact set. The following statements are equivalent:

(a) $\left(K, \tau_{p}\right)$ is fragmented by the norm.

(b) $\left.\overline{(\overline{\operatorname{span}(K)}}\|\|, \tau_{p}\right)$ is $\sigma$-fragmented by the norm.

(c) $(K, \gamma(D))$ is Lindelöf.

(d) $\left.\overline{(\operatorname{span}(K)}{ }^{\|} \|, \gamma(D)\right)$ is Lindelöf.

6. Banach spaces generated by Lindelöf subsets. In this section we study Banach spaces which are Lindelöf in the weak topology. Main tools are again the projectional generators. Beyond Theorem 6.1 below, which gives quite a general way of deciding when a Banach space is weakly Lindelöf, here we take advantage of the scope of the results in Section 4 and the main results in [4] to prove that a Banach space $X$ generated by a weakly Lindelöf subset which is $\sigma(X, F)$-compact with respect to some norming subspace $F \subset X^{*}$ is weakly Lindelöf. We need the following definition. For each set $\Gamma$, let $\Sigma(\Gamma)$ be the subspace of $\ell^{\infty}(\Gamma)$ consisting of all $u \in \ell^{\infty}(\Gamma)$ with $\{\gamma: u(\gamma) \neq 0\}$ at most countable. A compact Hausdorff space $K$ is said to be Corson if, for some $\Gamma, K$ can be embedded in $\Sigma(\Gamma)$ as a pointwise compact subset.

Definition 4 ([1]). A Banach space $X$ is said to be weakly Lindelöf determined (WLD for short) if there is a bounded one-to-one linear map $T: X^{*} \rightarrow \ell^{\infty}(\Gamma)$, for some set $\Gamma$, which is $\sigma\left(X^{*}, X\right)$-pointwise continuous and such that $T\left(X^{*}\right) \subset \Sigma(\Gamma)$

It was established in [26] that a Banach space is WLD if, and only if, its dual unit ball with the weak* topology is Corson compact. Note that WCG Banach spaces and hence separable Banach spaces are WLD. It is known that a WLD Banach space is $\gamma\left(B_{X^{*}}\right)$-Lindelöf $([25])$ and renormable by a LUR norm ([34] and [21]). A Banach space $X$, or more generally a convex subset $M$ of $X$, is said to have property $\mathcal{C}$ (after Corson) if each collection of relatively closed convex subsets of $M$ with empty intersection has a countable subcollection with empty intersection. If ( $M$, weak) is Lindelöf, then $M$ has property $\mathcal{C}$ since closed convex sets in $X$ are also weak-closed. It is shown in [28] that the Banach space $X$ has the property $\mathcal{C}$ if and only if, whenever $A \subset X^{*}$ and $f \in \bar{A}^{\mathrm{w}^{*}}$, there is a countable subset $C$ of $A$ 
such that $f \in \overline{\operatorname{co}(C)}{ }^{\text {w* }}$. This fact is crucial in the proof of the next theorem.

Theorem 6.1. Let $X$ be a Banach space with a projectional generator. If $X$ has property $\mathcal{C}$, then $X$ is $W L D$, i.e. $\left(B_{X^{*}}\right.$, weak $\left.k^{*}\right)$ is Corson compact.

Proof. Let $\varphi: F \rightarrow 2^{X}$ be a projectional generator on $X$, where $F$ is a norming subspace for $X$. Then $X$ admits a PRI constructed as we have recalled in Theorem 5.1. Let $\left\{P_{\alpha}: \omega_{0} \leq \alpha \leq \mu\right\}$ be this PRI. Since property $\mathcal{C}$ is stable under taking closed subspaces, each $P_{\alpha}(X)$ has property $\mathcal{C}$ and a projectional generator. Now, by a standard induction process on the density character of the Banach space, we may assume that $X$ admits a PRI $\left\{P_{\alpha}: \omega_{0} \leq \alpha \leq \mu\right\}$, with $\mu$ a limit ordinal, such that, for each $\omega_{0} \leq \alpha<\mu$, $P_{\alpha}(X)$ is WLD; that is, there is a one-to-one norm one operator

$$
T_{\alpha}: P_{\alpha}^{*}\left(X^{*}\right) \rightarrow \ell^{\infty}\left(\Gamma_{\alpha}\right) \quad \text { with } T_{\alpha}\left(P_{\alpha}^{*}\left(X^{*}\right)\right) \subset \Sigma\left(\Gamma_{\alpha}\right)
$$

which is weak ${ }^{*}$-pointwise continuous. Assume that $\left\{\Gamma_{\alpha}: \omega_{0} \leq \alpha<\mu\right\}$ is a disjoint family. Then we define

$$
\Gamma=\Gamma_{\omega_{0}} \cup \bigcup\left\{\Gamma_{\alpha+1}: \omega_{0} \leq \alpha<\mu\right\}
$$

and $T: X^{*} \rightarrow \ell^{\infty}(\Gamma)$ by the formulas

$$
\begin{aligned}
& (T f)(n)=T_{\omega_{0}}\left(P_{\omega_{0}}^{*}(f)\right)(n) \quad \text { if } n \in \Gamma_{\omega_{0}}=\mathbb{N}, \\
& (T f)(\gamma)=T_{\alpha+1}\left(P_{\alpha+1}^{*}(f)-P_{\alpha}^{*}(f)\right)(\gamma) \quad \text { if } \gamma \in \Gamma_{\alpha+1}, \omega_{0} \leq \alpha<\mu .
\end{aligned}
$$

Clearly $T$ is bounded linear and weak ${ }^{*}$-pointwise continuous. We claim that $T\left(X^{*}\right) \subset \Sigma(\Gamma)$. To prove it, we will see that the set $\left\{\alpha \in\left[\omega_{0}, \mu\right): P_{\alpha+1}^{*}(f)-\right.$ $\left.P_{\alpha}^{*}(f) \neq 0\right\}$ is at most countable for each $f \in X^{*}$. Assume on the contrary that this is not the case and take $f \in X^{*}$ so that this set is uncountable. Recall that the family $\left\{B_{\alpha}: \alpha<\mu\right\}$ is a long sequence of increasing $\mathbb{Q}$ linear subsets of $F$ with $P_{\alpha}^{*}\left(X^{*}\right)=\bar{B}_{\alpha}^{\mathrm{w}^{*}}$ for each $\alpha<\mu$. Also for each limit ordinal $\beta \leq \mu$ and $f \in X^{*}$, weak ${ }^{*}-\lim _{\alpha \uparrow \beta} P_{\alpha}^{*}(f)=P_{\beta}^{*}(f)$, and $P_{\mu}^{*}=$ Id. Let $\Delta=\left\{\alpha \in\left[\omega_{0}, \mu\right): P_{\alpha+1}^{*}(f)-P_{\alpha}^{*}(f) \neq 0\right\}$. Then $\Delta$ is an uncountable subset of $\left[\omega_{0}, \mu\right)$ which is well-ordered under the inherited ordering. Therefore there is an order-isomorphism $\varphi$ from $\left[0, \omega_{1}\right)$ onto an initial segment of $\Delta$. Let $\eta=\sup \varphi\left(\left[0, \omega_{1}\right)\right) \leq \mu$. Then $P_{\eta}^{*}(f)=$ weak $^{*}-\lim _{\gamma \uparrow \omega_{1}} P_{\varphi(\gamma)}^{*}(f)$ and therefore

$$
P_{\eta}^{*}(f) \in{\overline{\bigcup_{\gamma<\omega_{1}} P_{\varphi(\gamma)}^{*}(f)}}^{\mathrm{w}^{*}} .
$$

Since $X$ has property $\mathcal{C}$, there is a sequence $\gamma_{1}<\gamma_{2}<\ldots$ in $\left[0, \omega_{1}\right)$ such that

$$
P_{\eta}^{*}(f) \in \overline{\operatorname{co}\left(\bigcup_{i=1}^{\infty} P_{\varphi\left(\gamma_{i}\right)}^{*}(f)\right)} \mathrm{w}^{*} .
$$

Let $\xi=\varphi\left(\sup _{i} \gamma_{i}\right) \in \Delta$. Then $\xi<\eta \leq \mu$. Since for each $i, P_{\varphi\left(\gamma_{i}\right)}(f) \in$ $\bar{B}_{\varphi\left(\gamma_{i}\right)}^{\mathrm{w}^{*}} \subset \bar{B}_{\xi}^{\mathrm{w}^{*}}$, we have $P_{\eta}^{*}(f) \in \bar{B}_{\xi}^{\mathrm{w}^{*}}$. It follows that $P_{\eta}^{*}(f)$ is a fixed point of 
$P_{\alpha}^{*}$ for all $\alpha \geq \xi$. Hence if $\xi \leq \alpha<\eta$, then $P_{\eta}^{*}(f)=P_{\alpha}^{*} P_{\eta}^{*}(f)=P_{\alpha}^{*}(f)$ by the property of PRI: $P_{\eta} P_{\alpha}=P_{\min \{\eta, \alpha\}}$. In particular, $P_{\xi+1}^{*}(f)=P_{\eta}^{*}(f)=P_{\xi}^{*}(f)$, contradicting $\xi \in \Delta$. Hence $T\left(X^{*}\right) \subset \Sigma(\Gamma)$.

To see that $T$ is one-to-one, let $T(f)=0$ for an $f \in X^{*}$. Then $P_{\omega_{0}}^{*}(f)=0$, and $P_{\alpha+1}^{*}(f)=P_{\alpha}^{*}(f)=0$ for all $\alpha \in\left[\omega_{0}, \mu\right)$. Then by a straightforward (transfinite) induction, $P_{\alpha}^{*}(f)=0$ for all $\alpha \in\left[\omega_{0}, \mu\right)$, and hence $f=$ weak*$\lim _{\alpha \uparrow \mu} P_{\alpha}^{*}(f)=0$.

A combination of Theorems 5.5 and 6.1 gives us the following:

Corollary 6.2. Let $X$ be a Banach space, $F$ a norming subset of $X^{*}$, $H$ a bounded subset of $X$ which is $\sigma(X, F)$-compact fragmented by the norm of $X$, and let $Y=\overline{\operatorname{span}(H)}\|\|$. If $Y$ has property $\mathcal{C}$, then $Y$ is WLD.

As mentioned earlier, a WLD Banach space is weakly Lindelöf, but the converse is not true; cf. [21, p. 514]. In [21, p. 521], Mercourakis and Negrepontis have asked if this converse is true in dual Banach spaces. The affirmative answer to this question is contained in [25] where it is shown that if $X$ is an Asplund space then $X^{*}$ is weakly Lindelöf if and only if $\left(B_{X^{* *}}\right.$, weak $\left.^{*}\right)$ is Corson compact, i.e. $X^{*}$ is WLD. Recall that Edgar had observed earlier [9] that $X$ is an Asplund space whenever $X^{*}$ is weakly Lindelöf. The next two corollaries are generalizations of the result in [25] just mentioned. The first one is a special case of the previous corollary.

Corollary 6.3. Let $X$ be an Asplund space, $H$ a subset of $X^{*}$ which is weak ${ }^{*}$-compact, and let $Y=\overline{\operatorname{span}(H)}\|\|$. If $Y$ has property $\mathcal{C}$ then $Y$ is $W L D$. In particular, if $X$ is an Asplund space, then $X^{*}$ is WLD if and only if it has property $\mathcal{C}$.

A combination of most of the results in this paper and the main result in [4] finally allows us to prove:

Corollary 6.4. Let $X$ be a Banach space, and let $H$ be a subset of $X^{*}$ which is weak ${ }^{*}$-compact and weakly Lindelöf. Then the space generated by $H, Y=\overline{\operatorname{span}(H)}\|\|$, is WLD. In particular, $Y$ is weakly Lindelöf.

Proof. By the remark following Theorem 4.5, we know that the weak*closed absolute convex hull of $H$, say $W$, is also weakly Lindelöf. Hence by Corollary E of [4], ( $W$, weak) is fragmented by the norm. Furthermore, $Y=\overline{\operatorname{span}(W)}^{\|}\left\|={\overline{\bigcup_{n=1}^{\infty} n W}}^{\|}\right\|$has property $\mathcal{C}$ by Proposition 2 in [28]. Hence by Corollary 6.2, $Y$ is WLD, and since a closed subspace of a WLD Banach space is again WLD ([21]), the corollary follows.

REMARK 6.5. Let us remark that the statement that $Y$ is weakly Lindelöf in the previous corollary can be proved more directly using Proposition 4.1, Corollary 5.6 and Theorem 4.5. With the notation above we know 
that $Z:=\operatorname{span}(H)=\bigcup_{n=1}^{\infty} n W$ is weakly Lindelöf, because it is a countable union of weakly Lindelöf subsets. Therefore by Proposition 4.1, $\gamma\left(X^{*}, X\right)$ is stronger than the weak topology on $Z$. On the other hand, $Y=\bar{Z}^{\|\|}$is Lindelöf with respect to $\gamma\left(X^{*}, X\right)$ by Corollary 5.6, since $H$ is weak ${ }^{*}$-compact and $(H$, weak) is fragmented by the norm. Consequently, $Y$ will be weakly Lindelöf if we can prove that $\gamma\left(X^{*}, X\right)$ is stronger than the weak topology on $Y$. For this, it is sufficient to prove that for each $x^{* *} \in X^{* *}$ the restriction $\left.x^{* *}\right|_{Y}$ is $\gamma\left(X^{*}, X\right)$-continuous. We know from the above that $\left.x^{* *}\right|_{Z}$ is $\gamma\left(X^{*}, X\right)$-continuous. This means that for each $\varepsilon>0$ there is a $\gamma\left(X^{*}, X\right)$ open neighborhood $U \subset X^{*}$ of the origin such that

$$
\left|x^{* *}(g)\right|<\varepsilon \quad \text { for each } g \in U \cap Z \text {. }
$$

Now $U$ is also \|\| -open and therefore $\overline{U \cap Z}\|\|=\overline{U \cap Y}^{\|\|} \supset U \cap Y$. Therefore the \|\| -continuity of $x^{* *}$ and (14) imply that $\left|x^{* *}(f)\right| \leq \varepsilon$ for every $f \in U \cap Y$. This means that $x^{* *}$ is $\gamma\left(X^{*}, X\right)$-continuous on $Y$, which concludes the proof.

7. Examples of spaces generated by RN-compact subsets. As mentioned at the beginning of Section 5, in this section we give several examples of Banach spaces generated by an RN-compact subset. By Theorem 5.5, these spaces share all the properties stated in Theorem 5.1. Also, by Corollary 6.2 , for these spaces being WLD is equivalent to having property $\mathcal{C}$.

EXAMPLE A: Spaces with 1-norming Markushevich basis. Let us recall that a Markushevich basis, or M-basis, of a Banach space $X$ is a subset $\left\{\left(x_{i}, f_{i}\right): i \in I\right\}$ of $X \times X^{*}$ such that
(a) ${\overline{\operatorname{span}\left\{x_{i}: i \in I\right\}}}^{\|\|}=X$.
(b) $\bigcap_{i \in I} \operatorname{Kernel}\left(f_{i}\right)=\{0\}$.
(c) $f_{j}\left(x_{i}\right)=\delta_{i j}, i, j \in I$.

Let us consider the subspace $F:=\operatorname{span}\left\{f_{i}\right\}$, which is a total subspace in $X^{*}$ by condition (b). If $K:=\left\{x_{i}: i \in I\right\} \cup\{0\}$ then it is easy to see that $K$ is a $\sigma(X, F)$-compact set fragmented by the norm. Indeed, $\left\{x_{i}: i \in I\right\}$ is a $\sigma(X, F)$-discrete set with 0 as its unique limit point. When $F$ is norming, the M-basis is called a norming M-basis. Therefore any Banach space with a norming M-basis is generated by an RN-compact subset. The $\sigma$ fragmentability of spaces with a norming M-basis was first proved in [17]; here, it is a consequence of Theorem 5.8.

ExAmple B: Spaces of continuous functions. Let $K$ be a compact space and $D$ a dense subset of $K$. If $H \subset C(K)$ is $\tau_{p}(D)$-compact, uniformly bounded, fragmented by the supremum norm and separates the points of $K$, 
then $C(K)$ is generated by an $\mathrm{RN}$-compact set. Indeed, in this case the norming subspace of $C(K)^{*}$ is $F=\operatorname{span}\left\{\delta_{x}: x \in D\right\}$ and we observe that for every $n=1,2, \ldots$ the set

$$
H^{n}:=\left\{f_{1} \cdot \ldots \cdot f_{n}: f_{i} \in H, i=1, \ldots, n\right\}
$$

is $\sigma(C(K), F)$-compact and fragmented by the norm in view of Lemmas 2.1 and 2.2 in [24]. Now, $W=\bigcup_{n=1}^{\infty}(1 / n) H^{n} \cup\{0\}$ is also $\sigma(C(K), F)$ compact and $\sigma$-fragmented by the norm, hence fragmented [16, Theorem 4.1]. On the other hand, the Stone-Weierstrass theorem gives us the equality $\overline{\operatorname{span}(W)}^{\|\|}=C(K)$ and so $C(K)$ is generated by a $\sigma(C(K), F)$-compact subset fragmented by the norm.

EXAmPle C: Spaces of continuous functions defined on solid compact spaces and on compact spaces defined through adequate families of sets. Let $I$ be a set and consider the cube $[0,1]^{I}$ with the product topology. Given $x \in[0,1]^{I}$ let us write

$$
\begin{aligned}
\operatorname{supp}(x) & :=\{i \in I: x(i) \neq 0\}, \\
\mathcal{F}(I) & :=\left\{x \in[0,1]^{I}: \operatorname{supp}(x) \text { is finite }\right\} .
\end{aligned}
$$

We claim that if $K \subset[0,1]^{I}$ is a compact subset such that $K \cap \mathcal{F}(I)$ is dense in $K$ (i.e. $K$ is a special type of Valdivia compact space), then $C(K)$ is generated by an RN-compact subset. Indeed, write $D=K \cap \mathcal{F}(I)$ and let $\pi_{i}:[0,1]^{I} \rightarrow[0,1]$ denote the canonical projection onto the $i$-th coordinate, for each $i \in I$. Without loss of generality we can, and do, assume that for each $i \in I$ there is $x \in K$ such that $\pi_{i}(x) \neq 0$, because otherwise we can remove from the index set $I$ the element $i$ that is not needed for embedding $K$ in $[0,1]^{I}$. Observe that $\left\{\pi_{i}: i \in I\right\}$ is $\tau_{p}(D)$-discrete and that each $\tau_{p}(D)$ neighborhood of 0 must contain all but at most finitely many $\left\{\pi_{i}: i \in I\right\}$; therefore $\left\{\pi_{i}: i \in I\right\} \cup\{0\}$ is $\tau_{p}(D)$-compact, \|\|$_{\infty}$-fragmented and separates the points of $K$. We now use Example B to conclude that $C(K)$ is generated by an RN-compact subset.

A compact space $K \subset[0,1]^{I}$ is said to be solid if whenever $x \in K$ and $y \in[0,1]^{I}$ are such that either $y_{i}=x_{i}$ or $y_{i}=0$, for every $i \in I$, then $y \in K$. Obviously, if $K \subset[0,1]^{I}$ is solid, then $K \cap \mathcal{F}(I)$ is dense in $K$ and therefore $C(K)$ is generated by an $\mathrm{RN}$-compact set in view of our former reasoning.

A particular situation to which we can apply the above is when we deal with compact spaces defined through adequate families of sets. Following Talagrand [33], if $I$ is a non-empty set, a family $\mathcal{A}$ of subsets of $I$ is called adequate if it has the following properties:

(a) If $A \in \mathcal{A}$ and $B \subset A$, then $B \in \mathcal{A}$.

(b) $\{i\} \in \mathcal{A}$ for every $i \in I$.

(c) If $A \subset I$ and every finite subset of $A$ belongs to $\mathcal{A}$, then $A \in \mathcal{A}$. 
If $\mathcal{A}$ is an adequate family in $I$, then

$$
K:=\left\{\chi_{A}: A \in \mathcal{A}\right\}
$$

is a solid compact space. Then $C(K)$ is also generated by an RN-compact subset. Talagrand produced in [33, Théorème 4.3] an example of a compact space $K$ defined through an adequate family of sets that is not Eberlein compact; the corresponding $C(K)$ then does not contain a $\tau_{p}(K)$-compact subset separating the points of $K$, even though it contains a $\tau_{p}(D)$-compact (for a certain dense $D \subset K)\|\|_{\infty}$-fragmented subset separating the points of $K$.

Example D: Spaces of Bochner integrable functions. Let $(X,\|\|)$ be a Banach space and $F \subset X^{*}$ a norming subspace. It was stated in [6, Corollary 4.3 ] that if $\sigma(X, F)$-separable compact subsets of $X$ are \|\| -separable then $\sigma(X, F)$-compact (norm-bounded) subsets $H$ of $X$ are \|\| -fragmented. This is indeed a consequence of the equivalence between the first two statements in Theorem 2.1: write $D=F \cap B_{X^{*}}$ and consider $H \subset[-1,1]^{D}$; for $A \subset$ $D$ countable the set $\left.H\right|_{A} \subset[-1,1]^{A}$ is compact and metrizable, therefore separable; then there is a $\sigma(X, F)$-compact and separable $S \subset H$ such that $\left.S\right|_{A}=\left.H\right|_{A}$; the restriction map $[-1,1]^{D} \rightarrow[-1,1]^{A}$ is continuous for the corresponding uniform metrics and therefore $\left.H\right|_{A}$ is $d_{A}$-separable, because $S$ is $d_{D}$-separable ( $S$ is \|\| -separable).

The above observation is useful in finding more compact spaces "living" in Banach spaces and fragmented by the norm without being necessarily weakly compact.

Given a probability space $(\Omega, \Sigma, \mu)$ we will denote by $L^{p}(\mu, X), 1 \leq$ $p<\infty$, the Banach space of $\mu$-strongly measurable $X$-valued $p$-Bochner integrable functions $f: \Omega \rightarrow X$ normed by

$$
\|f\|_{p}=\left(\int_{\Omega}\|f\|^{p} d \mu\right)^{1 / p} .
$$

The dual $L^{p}(\mu, X)^{*}$ of $L^{p}(\mu, X)$ is a space of weak ${ }^{*}$-measurable functions and the space $L^{q}\left(\mu, X^{*}\right), 1=1 / p+1 / q$, which can be isometrically identified with a subspace of $L^{p}(\mu, X)^{*}$, is a norming subspace. So $\sigma^{\prime}=\sigma\left(L^{p}(\mu, X)\right.$, $\left.L^{q}\left(\mu, X^{*}\right)\right)$ is a Hausdorff topology which is weaker than the weak topology of $L^{p}(\mu, X)$; these two topologies coincide if, and only if, $X^{*}$ has the RNP [8, IV.1.1]. It was shown in [6, Example E] that every $\sigma^{\prime}$-separable compact subset of $L^{p}(\mu, X)$ is norm-separable. Therefore, every $\sigma^{\prime}$-compact subset of $L^{p}(\mu, X)$ is fragmented by the norm. Thus we can apply the results in Sections 5 and 6 to state for instance that if $H \subset L^{p}(\mu, X)$ is $\sigma^{\prime}$-compact then the space $Y=\overline{\operatorname{span}(H)}\|\|_{p}$ has a PRI. This result is related to the main result of [3], which asserts the existence of a bounded one-to-one operator from $\overline{\operatorname{span}(H)} \sigma^{\prime}$ into some $c_{0}(\Gamma)$ which is $\sigma^{\prime}$-pointwise continuous. 


\section{References}

[1] S. Argyros and S. Mercourakis, On weakly Lindelöf Banach spaces, Rocky Mountain J. Math. 23 (1993), 395-446.

[2] A. V. Arkhangel'skiı̌, Topological Function Spaces, Kluwer, Dordrecht, 1992.

[3] J. Batt and G. Schlüchtermann, Eberlein compacts in $L_{1}(X)$, Studia Math. 83 (1986), 239-250.

[4] B. Cascales, I. Namioka and G. Vera, The Lindelöf property and fragmentability, Proc. Amer. Math. Soc. 128 (2000), 3301-3309.

[5] B. Cascales and J. Orihuela, On pointwise and weak compactness in spaces of continuous functions, Bull. Soc. Math. Belg. Sér. B 40 (1988), 331-352.

[6] B. Cascales and G. Vera, Topologies weaker than the weak topology of a Banach space, J. Math. Anal. Appl. 182 (1994), 41-68.

[7] R. Deville, G. Godefroy and V. Zizler, Smoothness and Renormings in Banach Spaces, Pitman Monographs Surveys Pure Appl. Math. 64, Longman, 1993.

[8] J. Diestel and J. Uhl, Vector Measures, Math. Surveys 15, Amer. Math. Soc., Providence, RI, 1977.

[9] G. A. Edgar, Measurability in a Banach space, Indiana Univ. Math. J. 26 (1977), 663-677.

[10] R. Engelking, General Topology, PWN-Polish Sci. Publ., Warszawa, 1977.

[11] M. Fabian, Gâteaux Differentiability of Convex Functions and Topology. Weak Asplund Spaces, Wiley, New York, 1997.

[12] G. Godefroy, Boundaries of a convex set and interpolation sets, Math. Ann. 277 (1987), 173-184.

[13] - Five lectures in geometry of Banach spaces, in: Seminar on Functional Analysis, 1987 (Murcia, 1987), Notes Mat. 1, Univ. Murcia, Murcia, 1988, 9-67.

[14] R. W. Hansell, On Borel mappings and Baire functions, Trans. Amer. Math. Soc. 194 (1974), 161-188 and 195-211.

[15] J. E. Jayne, I. Namioka and C. A. Rogers, $\sigma$-fragmentable Banach spaces, Mathematika 39 (1992), 197-215.

[16] —, - - - Topological properties of Banach spaces, Proc. London Math. Soc. 66 (1993), 651-672.

[17] —, —, 一, $\sigma$-fragmented Banach spaces II, Studia Math. 111 (1994), 69-80.

[18] J. E. Jayne, J. Orihuela, A. J. Pallarés and G. Vera, $\sigma$-fragmentability of multivalued maps and selection theorems, J. Funct. Anal. 117 (1993), 243-273.

[19] J. E. Jayne and C. A. Rogers, Borel selectors for upper semicontinuous set-valued maps, Acta Math. 155 (1985), 41-79.

[20] J. L. Kelley, General Topology, Springer, New York, 1975.

[21] S. Mercourakis and S. Negrepontis, Banach spaces and topology. II, in: Recent Progress in General Topology (Prague, 1991), North-Holland, Amsterdam, 1992, 493-536.

[22] P. R. Meyer, The Baire order problem for compact spaces, Duke Math. J. 33 (1966), 33-39.

[23] - Function spaces and the Aleksandrov-Urysohn conjecture, Ann. Mat. Pura Appl. (4) 86 (1970), 25-29.

[24] I. Namioka, Radon-Nikodým compact spaces and fragmentability, Mathematika 34 (1989), 258-281.

[25] J. Orihuela, On weakly Lindelöf Banach spaces, in: Progress in Functional Analysis (Peñíscola, 1990), North-Holland Math. Stud. 170, North-Holland, Amsterdam, 1992, 279-291. 
[26] J. Orihuela, W. Schachermayer and M. Valdivia, Every Radon-Nikodym Corson compact space is Eberlein compact, Studia Math. 98 (1991), 157-174.

[27] J. Orihuela and M. Valdivia, Projective generators and resolutions of identity in Banach spaces, Rev. Mat. Univ. Complut. Madrid 2 (1989), 179-199.

[28] R. Pol, On a question of H. H. Corson and some related problems, Fund. Math. 109 (1980), 143-154.

[29] C. A. Rogers, Functions of the first Baire class, J. London Math. Soc. (2) 37 (1988), $535-544$.

[30] Z. Semadeni, Banach Spaces of Continuous Functions. Vol. I, Monograf. Mat. 55, PWN-Polish Sci. Publ., Warszawa, 1971.

[31] S. Simons, A convergence theorem with boundary, Pacific J. Math. 40 (1972), 703708.

[32] M. Talagrand, Deux généralisations d'un théorème de I. Namioka, ibid. 81 (1979), 239-251.

[33] - Espaces de Banach faiblement $\mathcal{K}$-analytiques, Ann. of Math. 110 (1979), 407-438.

[34] M. Valdivia, Projective resolution of identity in $C(K)$ spaces, Arch. Math. (Basel) 54 (1990), 493-498.

Departamento de Matemáticas

Universidad de Murcia

30100 Espinardo Murcia, Spain

E-mail: beca@um.es

joseori@um.es
Department of Mathematics, Box 354350

University of Washington

Seattle, WA 98195-4350, U.S.A.

E-mail:namioka@math.washington.edu 